\title{
TOPICS IN NEUTRINO ASTROPHYSICS
}

\author{
W. C. HAXTON \\ Institute for Nuclear Theory, Box 351550, and Department of Physics, Box 351560 \\ University of Washington, Seattle, WA 98195, USA \\ E-mail: haxton@phys.washington.edu
}

\begin{abstract}
In these TASI summer school lectures I discuss three topics in neutrino astrophysics: the solar neutrino problem, stellar cooling by neutrino emission, and the role of neutrinos in the nucleosynthesis that occurs within core-collapse supernovae.
\end{abstract}

\section{Introduction}

Part of the interest in neutrino astrophysics has to do with the fascinating interplay between nuclear and particle physics issues - e.g., whether neutrinos are massive and undergo flavor oscillations, whether they have detectable electromagnetic moments, etc. - and astrophysical phenomena, such as the clustering of matter on large scales, the mechanisms responsible for the synthesis of nuclei, and the evolution of stars. The three lectures here are intended to illustrate this interplay. The first lecture reviews the solar neutrino problem which, along with the atmospheric neutrino problem, provides perhaps our strongest direct evidence that new physics lurks beyond the standard model. The second has to with the implications of neutrino properties - e.g., whether neutrinos are Dirac or Majorana particles - for stellar cooling. The final lecture describes the nucleosynthesis we think accompanies a supernova explosion, and why that synthesis is a delicate probe of neutrino oscillations.

These lectures as well of those from several other 1998 TASI speakers share a common subtheme: how the extraordinary technical revolution in astronomy and astrophysics has made the microphysics of the universe more relevant. It is the precise data coming from the new generation of great observatories - maps of the cosmic microwave spectrum, precision measurements of the products of big bang nucleosynthesis, measurements of the solar neutrino spectrum, Hubble Space Telescope (HST) abundance distributions from early, metalpoor stars, detection of gamma ray bursts from cosmological sources - that allow us to form the connections between observations and the underlying microphysics. This is the driving force that is making the field of nuclear and particle astrophysics of such interest to both senior physicists and new students entering the field. 


\section{Solar Neutrinos 1}

More than three decades ago Ray Davis, Jr. and his collaborators 3 constructed a 0.615 kiloton $\mathrm{C}_{2} \mathrm{Cl}_{4}$ radiochemical solar neutrino detector in the Homestake Gold Mine, one mile beneath Lead, South Dakota. Within a few years it was apparent that the number of neutrinos detected was considerably below the predictions of the standard solar model, that is, the standard theory of main sequence stellar evolution.

Today the results from the ${ }^{37} \mathrm{Cl}$ detector, which have become quite accurate due to 30 years of careful measurement, have been augmented by results from four other experiments, the SAGE and the Kamiokanda $\mathrm{E}$ and SuperKamiokande 6 water Cerenkov detectors. It now appears that the combined results are very difficult to explain — some have argued impossible - by any plausible change in the standard solar model (SSM). Thus most believe that the answer to the solar neutrino problem is new particle physics, most likely some effect like solar neutrino oscillations associated with massive neutrinos. With the recent news that SuperKamiokande sees direct evidence for $\nu_{\mu}$ oscillations in the azimuthal dependence of atmospheric neutrinos, it seems that we may be on the threshold of a major discovery.

The purpose of this first (and longest) lecture is to summarize the solar neutrino problem and the arguments that it represents new particle physics.

\subsection{The Standard Solar Model 8}

Solar models trace the evolution of the sun over the past 4.6 billion years of main sequence burning, thereby predicting the present-day temperature and composition profiles of the solar core that govern neutrino production. Standard solar models share four basic assumptions:

* The sun evolves in hydrostatic equilibrium, maintaining a local balance between the gravitational force and the pressure gradient. To describe this condition in detail, one must specify the equation of state as a function of temperature, density, and composition.

* Energy is transported by radiation and convection. While the solar envelope is convective, radiative transport dominates in the core region where thermonuclear reactions take place. The opacity depends sensitively on the solar composition, particularly the abundances of heavier elements.

* Thermonuclear reaction chains generate solar energy. The standard model predicts that over $98 \%$ of this energy is produced from the pp chain conversion of four protons into ${ }^{4} \mathrm{He}$ (see Fig. 1)

$$
4 p \rightarrow{ }^{4} \mathrm{He}+2 \mathrm{e}^{+}+2 \nu_{\mathrm{e}}
$$


with proton burning through the CNO cycle contributing the remaining $2 \%$. The sun is a large but slow reactor: the core temperature, $T_{c} \sim 1.5 \cdot 10^{7} \mathrm{~K}$, results in typical center-of-mass energies for reacting particles of $\sim 10 \mathrm{keV}$, much less than the Coulomb barriers inhibiting charged particle nuclear reactions. Thus reaction cross sections are small: in most cases, as laboratory measurements are only possible at higher energies, cross section data must be extrapolated to the solar energies of interest.

* The model is constrained to produce today's solar radius, mass, and luminosity. An important assumption of the standard model is that the sun was highly convective, and therefore uniform in composition, when it first entered the main sequence. It is furthermore assumed that the surface abundances of metals (nuclei with $\mathrm{A}>5$ ) were undisturbed by the subsequent evolution, and thus provide a record of the initial solar metallicity. The remaining parameter is the initial ${ }^{4} \mathrm{He} / \mathrm{H}$ ratio, which is adjusted until the model reproduces the present solar luminosity after 4.6 billion years of evolution. The resulting ${ }^{4} \mathrm{He} / \mathrm{H}$ mass fraction ratio is typically $0.27 \pm 0.01$, which can be compared to the big-bang value of $0.23 \pm 0.01$. Note that the sun was formed from previously processed material.

The model that emerges is an evolving sun. As the core's chemical composition changes, the opacity and core temperature rise, producing a $44 \%$ luminosity increase since the onset of the main sequence. The temperature rise governs the competition between the three cycles of the pp chain: the ppI cycle dominates below about $1.6 \cdot 10^{7} \mathrm{~K}$; the ppII cycle between $(1.7-2.3)$ $\cdot 10^{7} \mathrm{~K}$; and the ppIII above $2.4 \cdot 10^{7} \mathrm{~K}$. The central core temperature of today's SSM is about $1.55 \cdot 10^{7} \mathrm{~K}$.

The competition between the cycles determines the pattern of neutrino fluxes. Thus one consequence of the thermal evolution of our sun is that the ${ }^{8} \mathrm{~B}$ neutrino flux, the most temperature-dependent component, proves to be of relatively recent origin: the predicted flux increases exponentially with a doubling period of about 0.9 billion years.

A final aspect of SSM evolution is the formation of composition gradients on nuclear burning timescales. Clearly there is a gradual enrichment of the solar core in ${ }^{4} \mathrm{He}$, the ashes of the pp chain. Another element, ${ }^{3} \mathrm{He}$, is a sort of catalyst for the pp chain, being produced and then consumed, and thus eventually reaching some equilibrium abundance. The timescale for equilibrium to be established as well as the eventually equilibrium abundance are both sharply decreasing functions of temperature, and thus increasing functions of the distance from the center of the core. Thus a steep ${ }^{3} \mathrm{He}$ density gradient is established over time.

The SSM has had some notable successes. From helioseismology the sound 


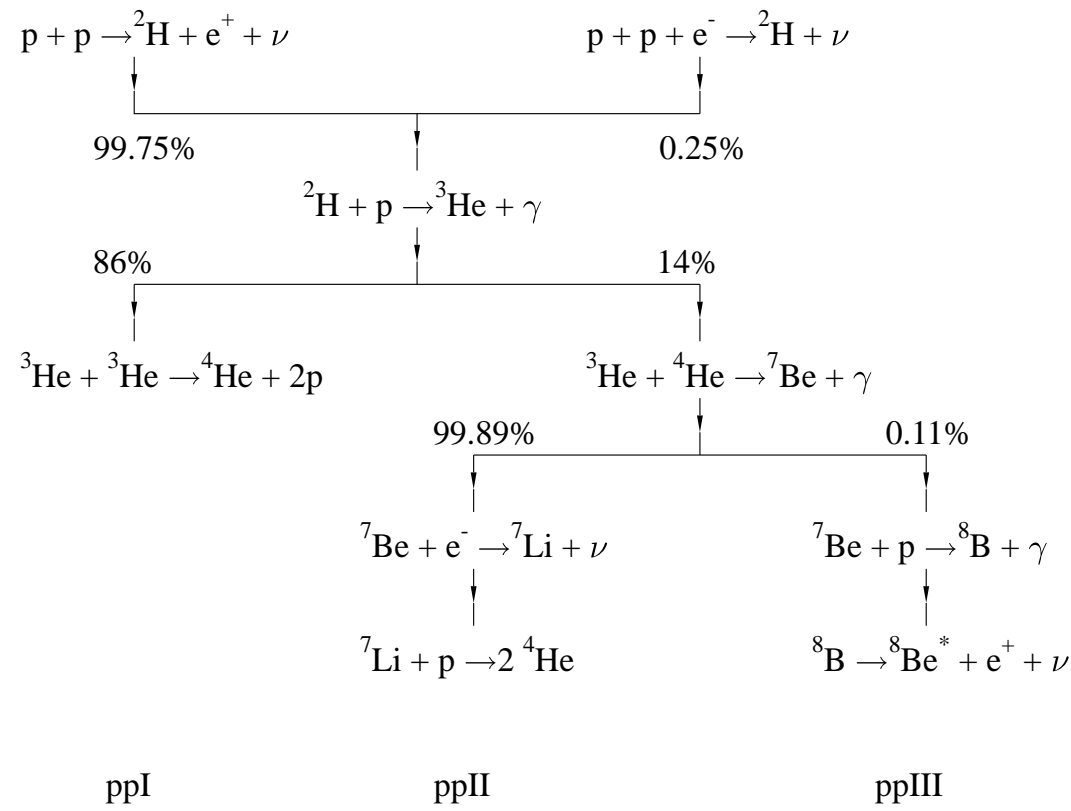

Figure 1: The solar pp chain.

speed profile $c(r)$ has been very accurately determined for the outer $90 \%$ of the sun, and is in excellent agreement with the SSM. Such studies verify important predictions of the SSM, such as the depth of the convective zone. However the SSM is not a complete model in that it does not explain all features of solar structure, such as the depletion of surface Li by two orders of magnitude. This is usually attributed to convective processes that operated at some epoch in our sun's history, dredging Li to a depth where burning takes place.

The principal neutrino-producing reactions of the pp chain and CNO cycle are summarized in Table 1 . The first six reactions produce $\beta$ decay neutrino spectra having allowed shapes with endpoints given by $\mathrm{E}_{\nu}^{\max }$. Deviations from an allowed spectrum occur for ${ }^{8} \mathrm{~B}$ neutrinos because the ${ }^{8} \mathrm{Be}$ final state is a broad resonance. The last two reactions produce line sources of electron capture neutrinos, with widths $\sim 2 \mathrm{keV}$ characteristic of the temperature of the solar core. Measurements of the pp, ${ }^{7} \mathrm{Be}$, and ${ }^{8} \mathrm{~B}$ neutrino fluxes will determine the relative contributions of the ppI, ppII, and ppIII cycles to solar energy generation. As discussed above, and as later illustrations will show more clearly, this competition is governed in large classes of solar models by 
Table 1: Solar neutrino sources and the flux predictions of the BP98 and Brun/TurckChieze/Morel SSMs in $\mathrm{cm}^{-2} \mathrm{~s}^{-1}$.

\begin{tabular}{|c|c|c|c|}
\hline Source & $\mathrm{E}_{\nu}^{\max }(\mathrm{MeV})$ & BP98 & BTCM98 \\
\hline $\mathrm{p}+\mathrm{p} \rightarrow{ }^{2} \mathrm{H}+\mathrm{e}^{+}+\nu$ & 0.42 & $5.94 \mathrm{E} 10$ & $5.98 \mathrm{E} 10$ \\
$13 \mathrm{~N} \rightarrow{ }^{13} \mathrm{C}+\mathrm{e}^{+}+\nu$ & 1.20 & $6.05 \mathrm{E} 8$ & $4.66 \mathrm{E} 8$ \\
${ }^{15} \mathrm{O} \rightarrow{ }^{15} \mathrm{~N}+\mathrm{e}^{+}+\nu$ & 1.73 & $5.32 \mathrm{E} 8$ & $3.97 \mathrm{E} 8$ \\
${ }^{17} \mathrm{~F} \rightarrow{ }^{17} \mathrm{O}+\mathrm{e}^{+}+\nu$ & 1.74 & $6.33 \mathrm{E} 6$ & \\
${ }^{8} \mathrm{~B} \rightarrow{ }^{8} \mathrm{Be}+\mathrm{e}^{+}+\nu$ & $\sim 15$ & $5.15 \mathrm{E} 6$ & $4.82 \mathrm{E} 6$ \\
${ }^{3} \mathrm{He}+\mathrm{p} \rightarrow{ }^{4} \mathrm{He}+\mathrm{e}^{+}+\nu$ & 18.77 & $2.10 \mathrm{E} 3$ & \\
${ }^{7} \mathrm{Be}+\mathrm{e}^{-} \rightarrow{ }^{7} \mathrm{Li}+\nu$ & $0.86(90 \%)$ & $4.80 \mathrm{E} 9$ & $4.70 \mathrm{E} 9$ \\
$\mathrm{p}+\mathrm{e}^{-}+\mathrm{p} \rightarrow{ }^{2} \mathrm{H}+\nu$ & $0.38(10 \%)$ & & \\
& 1.44 & $1.39 \mathrm{E} 8$ & $1.41 \mathrm{E} 8$ \\
\hline
\end{tabular}

a single parameter, the central temperature $T_{c}$. The flux predictions of the 1998 calculations of Bahfall, Basu, and Pinsonneault (BP98) and of Brun, Turck-Chieze and Morell 1 are included in Table 1.

\subsection{Solar Neutrino Detection 10}

Let us start with a brief reminder about low energy neutrino-nucleus interactions in detectors. Consider the charged current reaction

$$
\nu_{e}+(A, Z) \rightarrow e^{-}+(A, Z+1)
$$

Because the momentum transfer to the nucleus is very small for solar neutrinos, it can be neglected in the weak propagator, leading to an effective contact current-current interaction. If we begin with the simplest (though fictitious) case of the free neutron decay $n \rightarrow p$, the corresponding transition amplitude is then

$$
S_{f i}=\frac{G_{F}}{\sqrt{2}} \cos \theta_{C} \bar{u}(p) \gamma_{\mu}\left(1-g_{A} \gamma_{5}\right) u(n) \bar{u}(e) \gamma^{\mu}\left(1-\gamma_{5}\right) u(\nu)
$$

where $G_{F}$ is the weak coupling constant measured in muon decay and $\cos \theta_{c}$ gives the amplitude for the weak interaction to connect the $u$ quark to its firstgeneration partner, the d quark. The origin of this effective amplitude is the 
underlying standard model predictions for the elementary quark and lepton currents. The weak interactions at this level are predicted by the standard model to be exactly left handed. Experiment shows that the effective coupling of the $\mathrm{W}$ boson to the nucleon is governed by $\gamma_{\mu}\left(1-g_{A} \gamma_{5}\right)$, as noted above, where $g_{A} \sim 1.26$. The axial coupling is thus shifted from its underlying value by the strong interactions responsible for the binding of the quarks within the nucleon.

If an isolated nucleon were the target, one could proceed to calculate the cross section from the effective nucleon current given above. The extension to nuclear systems traditionally begins with the observation that nucleons in the nucleus are rather nonrelativistic, $v / c \sim 0.1$. The amplitude $\bar{u}(p) \gamma^{\mu}(1-$ $\left.g_{A} \gamma_{5}\right) u(n)$ can be expanded in powers of $p / M$. The leading vector and axial operators are readily found to be

$$
\begin{aligned}
\gamma_{0}: & 1 \\
\vec{\gamma}: & \vec{p} / M \sim v / c \\
\gamma_{0} \gamma_{5}: & \vec{\sigma} \cdot \vec{p} / M \sim v / c \\
\vec{\gamma} \gamma_{5}: & \vec{\sigma}
\end{aligned}
$$

Thus it is the time-like part of the vector current and the space-like part of the axial-vector current that survive in the nonrelativistic limit.

(In a nucleus these currents must be corrected for the presence of meson exchange contributions. The corrections to the vector charge and axial threecurrent, which we just pointed out survive in the nonrelativistic limit, are of order $(v / c)^{2} \sim 1 \%$. Thus the naive one-body currents are a very good approximation to the nuclear currents. In contrast, exchange current corrections to the axial charge and vector three-current operators are of order $v / c$, and thus of relative order 1 . This difficulty for the vector three-current can be largely circumvented, because current conservation as embodied in the generalized Siegert's theorem allows one to rewrite important parts of this operator in terms of the vector charge operator. In the long-wavelength limit appropriate to solar neutrinos, all terms unconstrained by current conservation do not survive. In effect, one has replaced a current operator with large two-body corrections by a charge operator with only small corrections. In contrast, the axial charge operator is significantly altered by exchange currents even for long-wavelength processes like $\beta$ decay. Typical axial-charge $\beta$ decay rates are enhanced by $\sim 2$ because of exchange currents.)

If such a nonrelativistic reduction is done for our single current one obtains

$$
S_{f i} \sim \cos \theta_{c} \frac{G_{F}}{\sqrt{2}}\left(\phi^{\dagger}(p) \phi(n) \bar{u}(e) \gamma^{0}\left(1-\gamma_{5}\right) u(\nu)\right.
$$




$$
\left.-\phi^{\dagger}(p) g_{A} \vec{\sigma} \phi(n) \cdot \bar{u}(e) \vec{\gamma}\left(1-\gamma_{5}\right) u(\nu)\right)
$$

where the $\phi$ are now two-component Pauli spinors for the nucleons. The above result can be generalized to include $\bar{\nu}_{e}$ reactions by introducing the isospin operators $\tau_{ \pm}$where $\tau_{+}|\mathrm{n}\rangle=|\mathrm{p}\rangle$ and $\tau_{-}|\mathrm{p}\rangle=|\mathrm{n}\rangle$, with all other matrix elements being zero. Thus

$$
\begin{gathered}
\phi^{\dagger}(p) \phi(n) \rightarrow \phi^{\dagger}(N) \tau_{ \pm} \phi(N) \\
\phi^{\dagger}(p) \vec{\sigma} \phi(n) \rightarrow \phi^{\dagger}(N) \vec{\sigma} \tau_{ \pm} \phi(N) .
\end{gathered}
$$

This result easily generalizes to nuclear decay. Given our comments about exchange currents, the first step is the replacement

$$
\begin{gathered}
\tau_{ \pm} \rightarrow \sum_{i=1}^{A} \tau_{ \pm}(i) \\
\sigma \tau_{ \pm} \rightarrow \sum_{i=1}^{A} \sigma(i) \tau_{ \pm}(i) .
\end{gathered}
$$

Plugging $S_{f i}$ into the standard cross section formula (which involves an average over initial and sum over final nuclear spins of the square of the transition amplitude) then yields the allowed nuclear matrix element

$$
\frac{1}{2 J_{i}+1}\left(\left|\left\langle f|| \sum_{i=1}^{A} \tau_{ \pm}(i)|| i\right\rangle\right|^{2}+g_{A}^{2}\left|\left\langle f|| \sum_{i=1}^{A} \sigma(i) \tau_{ \pm}(i) \| i\right\rangle\right|^{2}\right) .
$$

Our initial calculation for the nucleon treated that particle as structureless. Implicitly we assumed that the momentum transfer is much smaller than the inverse nucleon size. If we take $10 \mathrm{MeV}$ as a typical solar neutrino momentum transfer, these quantities would be in the ratio 1:20. For a light nucleus, the corresponding result might be 1:10. This long-wavelength approximation in combination with the nonrelativistic approximation yields the allowed result, where only Fermi and Gamow-Teller operators survive. These are the spinindependent and spin-dependent operators appearing above.

The Fermi operator is proportional to the isospin raising/lowering operator: in the limit of good isopsin, which typically is good to $5 \%$ or better in the description of low-lying nuclear states, it can only connect states in the same isospin multiplet, that is, states with a common spin-spatial structure. If the initial state has isospin $\left(T_{i}, M_{T i}\right)$, this final state has $\left(T_{i}, M_{T i} \pm 1\right)$ for $\left(\nu, e^{-}\right)$ and $\left(\bar{\nu}, e^{+}\right)$reactions, respectively, and is called the isospin analog state (IAS). 
In the limit of good isospin the sum rule for this operator in then particularly simple

$$
\sum_{f} \frac{1}{2 J_{i}+1}\left|\left\langle f|| \sum_{i=1}^{A} \tau_{+}(i) \| i\right\rangle\right|^{2}=\frac{1}{2 J_{i}+1}\left|\left\langle I A S|| \sum_{i=1}^{A} \tau_{+}(i) \| i\right\rangle\right|^{2}=|N-Z| .
$$

The excitation energy of the IAS relative to the parent ground state can be estimated accurately from the Coulomb energy difference

$$
E_{I A S} \sim\left(\frac{1.728 Z}{1.12 A^{1 / 3}+0.78}-1.293\right) \mathrm{MeV} .
$$

The angular distribution of the outgoing electron for a pure Fermi $(N, Z)+\nu \rightarrow$ $(N-1, Z+1)+e^{-}$transition is $1+\beta \cos \theta_{\nu e}$, and thus forward peaked. Here $\beta$ is the electron velocity.

The Gamow-Teller (GT) response is more complicated, as the operator can connect the ground state to many states in the final nucleus. In general we do not have a precise probe of the nuclear GT response apart from weak interactions themselves. However a good approximate probe is provided by forward-angle $(\mathrm{p}, \mathrm{n})$ scattering off nuclei, a technique that has been developed in particular by experimentalists at the Indiana University Cyclotron Facility. The $(\mathrm{p}, \mathrm{n})$ reaction transfers isospin and thus is superficially like $\left(\nu, e^{-}\right)$. At forward angles $(\mathrm{p}, \mathrm{n})$ reactions involve negligible three-momentum transfers to the nucleus. Thus the nucleus should not be radially excited. It thus seems quite plausible that forward-angle $(\mathrm{p}, \mathrm{n})$ reactions probe the isospin and spin of the nucleus, the macroscopic quantum numbers, and thus the Fermi and GT responses. For typical transitions, the correspondence between $(\mathrm{p}, \mathrm{n})$ and the weak GT operators is believed to be accurate to about $10 \%$. Of course, in a specific transition, much larger discrepancies can arise.

The (p,n) studies demonstrate that the GT strength tends to concentrate in a broad resonance centered at a position $\delta=E_{G T}-E_{I A S}$ relative to the IAS given by

$$
\delta \sim\left(7.0-28.9 \frac{N-Z}{A}\right) \mathrm{MeV} .
$$

Thus while the peak of the GT resonance is substantially above the IAS for $N \sim Z$ nuclei, it drops with increasing neutron excess. Thus $\delta \sim 0$ for $\mathrm{Pb}$. A typical value for the full width at half maximum $\Gamma$ is $\sim 5 \mathrm{MeV}$.

The approximate Ikeda sum rule constrains the difference in the $\left(\nu, e^{-}\right)$ and $\left(\bar{\nu}, e^{+}\right)$strengths

$$
\sum_{f}\left(\left|M_{G T}^{f i}\left(\nu, e^{-}\right)\right|^{2}-\left|M_{G T}^{f i}\left(\bar{\nu}, e^{+}\right)\right|^{2}\right)=3(N-Z)
$$


where

$$
\left|M_{G T}^{f i}\left(\nu, e^{-}\right)\right|^{2}=\frac{1}{2 J_{i}+1}\left|\left\langle f\left\|\sum_{i=1}^{A} \sigma(i) \tau_{+}(i)\right\| i\right\rangle\right|^{2} .
$$

In many cases of interest in heavy nuclei, the strength in the $\left(\bar{\nu}, e^{+}\right)$direction is largely blocked. For example, in a naive $2 s 1 d$ shell model description of ${ }^{37} \mathrm{Cl}$, the $\mathrm{p} \rightarrow \mathrm{n}$ direction is blocked by the closed neutron shell at $\mathrm{N}=20$. Thus this relation can provide an estimate of the total $\beta^{-}$strength. Experiment shows that the $\beta^{-}$strength found in and below the GT resonance does not saturate the Ikeda sum rule, typically accounting for $\sim(60-70) \%$ of the total. Measured and shell model predictions of individual GT transition strengths tend to differ systematically by about the same factor. Presumably the missing strength is spread over a broad interval of energies above the GT resonance. This is not unexpected if one keeps in mind that the shell model is an approximate effective theory designed to describe the long wavelength modes of nuclei: such a model should require effective operators, renormalized from their bare values. Phenomenologically, the shell model seems to require $11 g_{A}^{\text {eff }} \sim 1.0$ as well as a small spin-tensor term $\left(\sigma \otimes Y_{2}(\hat{r})\right)_{J=1}$ of relative strength $\sim 0.1$.

The angular distribution of $\mathrm{GT}(N, Z)+\nu_{e} \rightarrow(N-1, Z+1)+e^{-}$reactions is $3-\beta \cos \theta_{\nu e}$, corresponding to a gentle peaking in the backward direction.

The above discussion of allowed responses can be repeated for neutral current processes such as $\left(\nu, \nu^{\prime}\right)$. The analog of the Fermi operator contributes only to elastic processes, where the standard model nuclear weak charge is approximately the neutron number. As this operator does not generate transitions, it is not yet of much interest for solar or supernova neutrino detection, though there are efforts to develop low-threshold detectors (e.g., cryogenic technologies) for recording the modest nuclear recoil energies. The analog of the GT response involves

$$
\left|M_{G T}^{f i}\left(\nu, \nu^{\prime}\right)\right|^{2}=\frac{1}{2 J_{i}+1}\left|\left\langle f\left\|\sum_{i=1}^{A} \sigma(i) \frac{\tau_{3}(i)}{2}\right\| i\right\rangle\right|^{2} .
$$

The operator appearing in this expression is familiar from magnetic moments and magnetic transitions, where the large isovector magnetic moment $\left(\mu_{v} \sim\right.$ 4.706) often leads to it dominating the orbital and isoscalar spin operators.

Finally, there is one purely leptonic reaction of great interest, since it is the reaction exploited by Kamiokande and SuperKamiokande. Electron neutrinos can scattered off electrons via both charged and neutral current reactions. The cross section calculation is straight forward and will not be repeated here. Two features of the result are of importance for our later discussions, however. 
Because of the neutral current contribution, heavy-flavor $\left(\nu_{\mu}\right.$ and $\left.\nu_{\tau}\right)$ also scatter off electrons, but with a cross section reduced by about a factor of seven at low energies. Second, for neutrino energies well above the electron rest mass, the scattering is sharply forward peaked. Thus this reaction allows one to exploit the position of the sun in separating the solar neutrino signal from a large but isotropic background.

As I mentioned earlier, the first experiment performed was one exploiting the reaction

$$
{ }^{37} \mathrm{Cl}\left(\nu, \mathrm{e}^{-}\right){ }^{37} \mathrm{Ar} .
$$

As the threshold for this reaction is $0.814 \mathrm{MeV}$, the important neutrino sources are the ${ }^{7} \mathrm{Be}$ and ${ }^{8} \mathrm{~B}$ reactions. The ${ }^{7} \mathrm{Be}$ neutrinos excite just the GT transition to the ground state, the strength of which is known from the electron capture lifetime of ${ }^{37} \mathrm{Ar}$. The ${ }^{8} \mathrm{~B}$ neutrinos can excite all bound states in ${ }^{37} \mathrm{Ar}$, including the dominant transition to the IAS residing at an excitation of $4.99 \mathrm{MeV}$. The strength of excite-state GT transitions can be determined from the $\beta$ decay ${ }^{37} \mathrm{Ca}\left(\beta^{+}\right){ }^{37} \mathrm{~K}$, which is the isospin mirror reaction to ${ }^{37} \mathrm{Cl}\left(\nu, e^{-}\right){ }^{37} \mathrm{Ar}$. The net result is that, for SSM fluxes, $78 \%$ of the capture rate should be due to ${ }^{8} \mathrm{~B}$ neutrinos, and $15 \%$ to ${ }^{7} \mathrm{Be}$ neutrinos. The measured capture rate 12 $2.56 \pm 0.16 \pm 0.16 \mathrm{SNU}\left(1 \mathrm{SNU}=10^{-36}\right.$ capture/atom $\left./ \mathrm{sec}\right)$ is about $1 / 3$ the standard model value.

Similar radiochemical experiments were done by the SAGE and GALLEX collaborations using a different target, ${ }^{71} \mathrm{Ga}$. The special properties of this target include its low threshold and an unusually strong transition to the ground state of ${ }^{71} \mathrm{Ge}$, leading to a large pp neutrino cross section (see Fig. 2). The experimental capture rates are $66 \pm 13 \pm 6$ and $76 \pm 8$ SNU for the SAGE and GALLEX detectors, respectively. The SSM prediction is about 130 SNU 13. Most important, since the pp flux is directly constrained by the solar luminosity in all steady-state models, there is a minimum theoretical value for the capture rate of $79 \mathrm{SNU}$, given standard model weak interaction physics. Note there are substantial uncertainties in the ${ }^{71} \mathrm{Ga}$ cross section due to ${ }^{7} \mathrm{Be}$ neutrino capture to two excited states of unknown strength. These uncertainties were greatly reduced by direct calibrations of both detectors using ${ }^{51} \mathrm{Cr}$ neutrino sources.

The remaining experiments, Kamiokande II/III and SuperKamiokande, exploited water Cerenkov detectors to view solar neutrinos event-by-event. Solar neutrinos scatter off electrons, with the recoiling electrons producing the Cerenkov radiation that is then recorded in surrounding phototubes. Thresholds are determined by background rates; SuperKamiokande is currently operating with a trigger at approximately six $\mathrm{MeV}$. The initial experiment, Kamiokande II/III, found a flux of ${ }^{8} \mathrm{~B}$ neutrinos of $(2.91 \pm 0.24 \pm 0.35)$. 


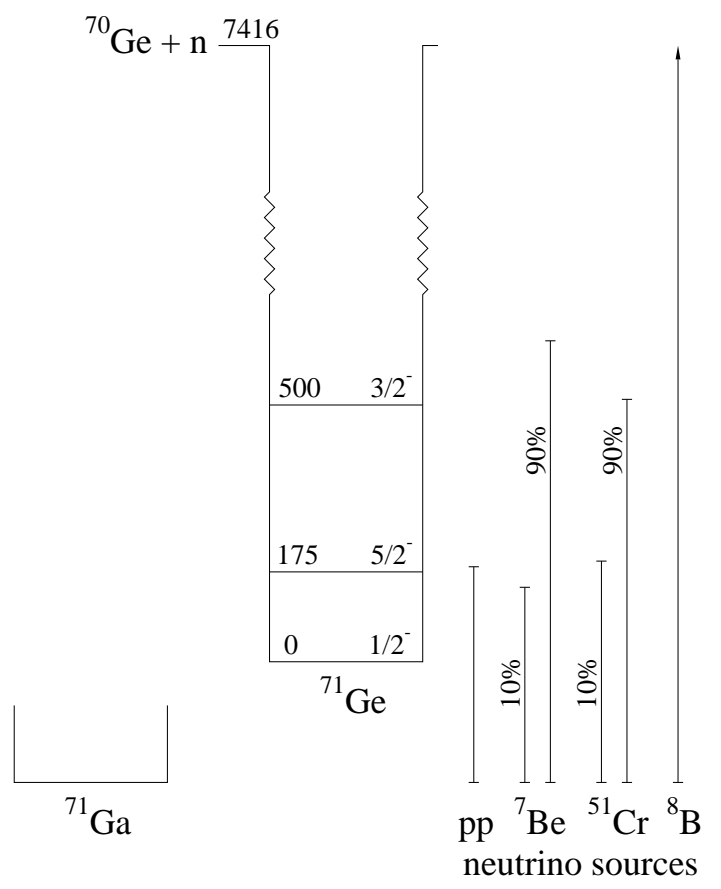

Figure 2: Level scheme for ${ }^{71} \mathrm{Ge}$ showing the excited states that contribute to absorption of $\mathrm{pp},{ }^{7} \mathrm{Be},{ }^{51} \mathrm{Crm}$ and ${ }^{8} \mathrm{~B}$ neutrinos.

$10^{6} / \mathrm{cm}^{2}$ s after about a decade of measurement. Its much larger successor SuperKamiokande, with a 22.5 kiloton fiducial volume, yielded the result $(2.37 \pm$ $0.06 \pm 0.08) \cdot 10^{6} / \mathrm{cm}^{2}$ s after the first 374 days of measurements. This is about $36 \%$ of the SSM flux. This result continues to improve in accuracy.

\subsection{Uncertainties in Standard Solar Model Parameters}

The pattern of solar neutrino fluxes that has emerged from these experiments is

$$
\begin{aligned}
\phi(p p) & \sim 0.9 \phi^{\mathrm{SSM}}(p p) \\
\phi\left({ }^{7} \mathrm{Be}\right) & \sim 0
\end{aligned}
$$




$$
\phi\left({ }^{8} \mathrm{~B}\right) \sim 0.43 \phi^{\mathrm{SSM}}\left({ }^{8} \mathrm{~B}\right) .
$$

A reduced ${ }^{8} \mathrm{~B}$ neutrino flux can be produced by lowering the central temperature of the sun somewhat, as $\phi\left({ }^{8} \mathrm{~B}\right) \sim T_{c}^{18}$. However, such an adjustment, either by varying the parameters of the SSM or by adopting some nonstandard physics, tends to push the $\phi\left({ }^{7} \mathrm{Be}\right) / \phi\left({ }^{8} \mathrm{~B}\right)$ ratio to higher values rather than the low one of eq. (12),

$$
\frac{\phi\left({ }^{7} \mathrm{Be}\right)}{\phi\left({ }^{8} \mathrm{~B}\right)} \sim T_{c}^{-10} .
$$

Thus the observations seem difficult to reconcile with plausible solar model variations: one observable $\left(\phi\left({ }^{8} \mathrm{~B}\right)\right)$ requires a cooler core while a second, the ratio $\phi\left({ }^{7} \mathrm{Be}\right) / \phi\left({ }^{8} \mathrm{~B}\right)$, requires a hotter one.

An initial question is whether this problem remains significant when one takes into account known uncertainties in the parameters of the SSM. While a detailed summary of standard model uncertainties would take us well beyond the limits of these lectures, a qualitative discussion of pp chain nuclear uncertainties is appropriate. This nuclear microphysics has been the focus of a great deal of experimental work. The pp chain involves a series of nonresonant charged-particle reactions occurring at center-of-mass energies that are well below the height of the inhibiting Coulomb barriers. As the resulting small cross sections preclude laboratory measurements at the relevant energies, one must extrapolate higher energy measurements to threshold to obtain solar cross sections. This extrapolation is often discussed in terms of the astrophysical S-factor

$$
\sigma(E)=\frac{S(E)}{E} \exp (-2 \pi \eta)
$$

where $\eta=\frac{Z_{1} Z_{2} \alpha}{\beta}$, with $\alpha$ the fine structure constant and $\beta=v / c$ the relative velocity of the colliding particles. This parameterization removes the gross Coulomb effects associated with the s-wave interactions of charged, point-like particles. The remaining energy dependence of $\mathrm{S}(\mathrm{E})$ is gentle and can be expressed as a low-order polynomial in E. Usually the variation of $\mathrm{S}(\mathrm{E})$ with $\mathrm{E}$ is taken from a direct reaction model and then used to extrapolate higher energy measurements to threshold. The model accounts for finite nuclear size effects, strong interaction effects, contributions from other partial waves, etc. As laboratory measurements are made with atomic nuclei while conditions in the solar core guarantee the complete ionization of light nuclei, additional corrections must be made to account for the different electronic screening environments.

Recently a large working group met at a workshop sponsored by the Institute for Nuclear Theory, University of Washington, to review past work on the 
nuclear reactions of the pp chain and $\mathrm{CNO}$ cycle, to recommend best values and appropriate errors, and to identify specific issues in experiment and theory where additional work is needed. The results will soon be published in Reviews of Modern Physics. I will not attempt a summary here, but will give one or two highlights.

The most significant recommend change involves the reaction ${ }^{7} \mathrm{Be}(\mathrm{p}, \gamma){ }^{8} \mathrm{~B}$, where the standard $\mathrm{S}_{17}(0) \sim 22.4 \mathrm{eVb}$ is that given 14 by Johnson et al. Measurements of $\mathrm{S}_{17}(\mathrm{E})$ are complicated by the need to use radioactive targets and thus to determine the areal density of the ${ }^{7} \mathrm{Be}$ target nuclei. Two techniques have been employed, measuring the rate of $478 \mathrm{keV}$ photons from ${ }^{7}$ Be decay or counting the daughter ${ }^{7} \mathrm{Li}$ nuclei via the reaction ${ }^{7} \mathrm{Li}(\mathrm{d}, \mathrm{p}){ }^{8} \mathrm{Li}$. The low-energy data sets for $\mathrm{S}_{17}(\mathrm{E})$ disagree by $25 \%$. This is a systematic normalization problem as each data set is consistent with theory in its dependence on E. The energy dependence below $\sim 500 \mathrm{keV}$ is believed to be quite simple as it is determined by the asymptotic nuclear wave function.

The Seattle working group on $\mathrm{S}_{17}(\mathrm{E})$ found that only one low-energy data set, that of Filippone et al. 15 , was described in the published literature in sufficient detail to be evaluated. The target activity in that experiment had been measured by both $478 \mathrm{keV}$ gamma rays and by the (d,p) reaction, with consistent results. The resulting recommended value was thus based on this measurement, yielding

$$
S_{17}(0)=19_{-2}^{+4} \mathrm{eV} \mathrm{b}, \quad 1 \sigma .
$$

The ${ }^{3} \mathrm{He}(\alpha, \gamma){ }^{7}$ Be reaction has been measured by two techniques, by counting the capture $\gamma$ rays and by detecting the resulting ${ }^{7} \mathrm{Be}$ activity. While the two techniques have been used by several groups and have yielded separately consistent results, the capture $\gamma$ ray value $\mathrm{S}_{17}(0)=0.507 \pm 0.016 \mathrm{keV}$ b is not in good agreement with the ${ }^{7} \mathrm{Be}$ activity value $0.572 \pm 0.026 \mathrm{keV}$-b. The Seattle working group concluded that the evidence for a systematic discrepancy of unknown origin was reasonably strong and recommended that standard procedures be used in assigning a suitably expanded error. The recommended value $\mathrm{S}_{34}(0)$ is $0.53 \pm 0.05$.

These and other recommended values were recently incorporated into the BP98 solar model calculation. While the workshop's recommended values involve no qualitative changes, there is some broadening of error bars. The downward shift in $\mathrm{S}_{17}(0)$ leads to a lower ${ }^{8} \mathrm{~B}$ flux. The workshop's Reviews of Modern Physics article summarizes a substantial amount of work on topics not discussed here: screening effects, weak radiative corrections to and exchange current effects on $\mathrm{p}+\mathrm{p}$, the atomic physics of ${ }^{7} \mathrm{Be}+\mathrm{e}^{-}$, etc. Much of this discussion was useful in evaluating possible uncertainties in solar microphysics, 
and in identifying opportunities for reducing these uncertainities.

Are uncertainties in the parameters of the SSM a significant source of uncertainty? The S-factors discussed above comprise one set of parameters, but there are others: the solar lifetime, the opacities, the solar luminosity, etc. In order to answer this question while also taking into account correlations among the fluxes when input parameters are varied, first Bahcall and Ulrich 16 and later Bahcall and Haxton 17 constructed 1000 SSMs by randomly varying five input parameters, the primordial heavy-element-to-hydrogen ratio $\mathrm{Z} / \mathrm{X}$ and $\mathrm{S}(0)$ for the p-p, ${ }^{3} \mathrm{He}-{ }^{3} \mathrm{He},{ }^{3} \mathrm{He}-{ }^{4} \mathrm{He}$, and $\mathrm{p}-{ }^{7} \mathrm{Be}$ reactions, assuming for each parameter a normal distribution with the mean and standard deviation. (These were the parameters assigned the largest uncertainties.) Smaller uncertainties from radiative opacities, the solar luminosity, and the solar age were folded into the results of the model calculations perturbatively.

The resulting pattern of ${ }^{7} \mathrm{Be}$ and ${ }^{8} \mathrm{~B}$ flux predictions is shown in Fig. 3. The elongated error ellipses indicate that the fluxes are strongly correlated. Those variations producing $\phi\left({ }^{8} \mathrm{~B}\right)$ below $0.8 \phi^{\mathrm{SSM}}\left({ }^{8} \mathrm{~B}\right)$ tend to produce a reduced $\phi\left({ }^{7} \mathrm{Be}\right)$, but the reduction is always less than 0.8 . Thus a greatly reduced $\phi\left({ }^{7} \mathrm{Be}\right)$ cannot be achieved within the uncertainties assigned to parameters in the SSM.

A similar exploration, but including parameter variatipns very far from their preferred values, was carried out by Castellani et al. 18, who displayed their results as a function of the resulting core temperature $T_{c}$. The pattern that emerges is striking (see Fig. 4): parameter variations producing the same value of $T_{c}$ produce remarkably similar fluxes. Thus $T_{c}$ provides an excellent one-parameter description of standard model perturbations. Figure 4 also illustrates the difficulty of producing a low ratio of $\phi\left({ }^{7} \mathrm{Be}\right) / \phi\left({ }^{8} \mathrm{~B}\right)$ when $T_{c}$ is reduced.

The 1000-solar-model variations were made under the constraint of reproducing the solar luminosity. Those variations show a similar strong correlation with $T_{c}$

$$
\phi(p p) \propto T_{c}^{-1.2} \quad \phi\left({ }^{7} \mathrm{Be}\right) \propto T_{c}^{8} \quad \phi\left({ }^{8} \mathrm{~B}\right) \propto T_{c}^{18} .
$$

Figures 3 and 4 offer a strong argument that reasonable variations in the parameters of the SSM, or nonstandard changes in quantities like the metallicity, opacities, or solar age, cannot produce the pattern of fluxes deduced from experiment (eq. (12)). This would seem to limit possible solutions to errors either in the underlying physics of the SSM or in our understanding of neutrino properties. 


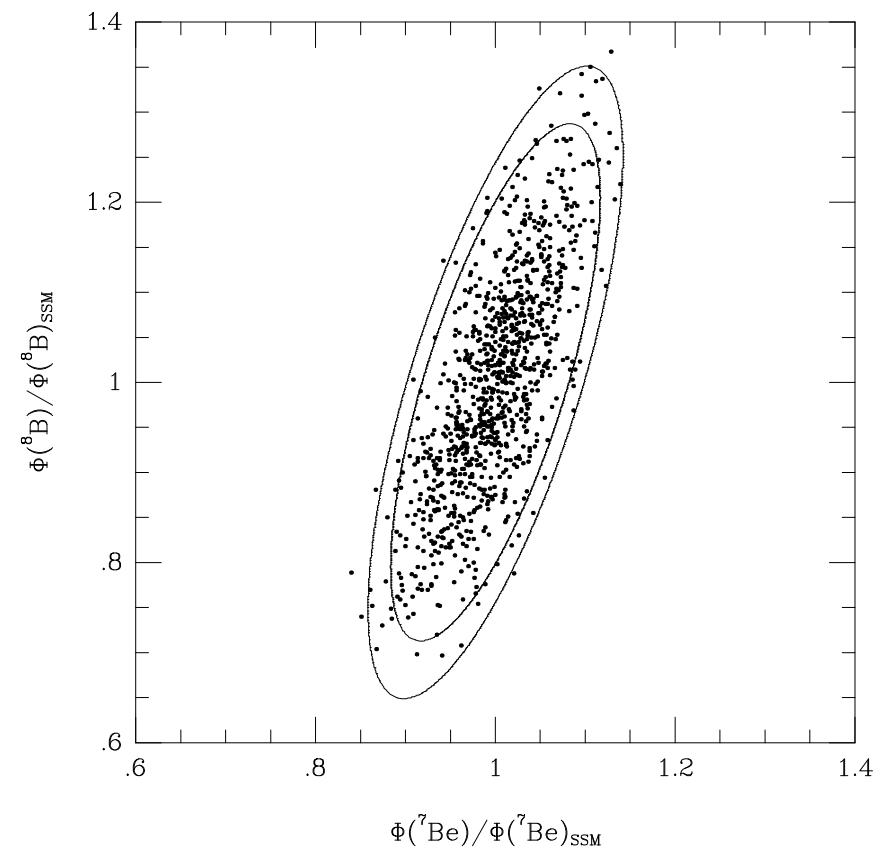

Figure 3: SSM ${ }^{7} \mathrm{Be}$ and ${ }^{8} B$ flux predictions. The dots represent the results of SSM calculations where the input parameters were varied according to their assigned uncertainties, as described in the text. The $90 \%$ and $99 \%$ confidence level error ellipses are shown.

\subsection{Nonstandard Solar Models}

Nonstandard solar models include both variations of SSM parameters far outside the ranges that are generally believed to be reasonable (some examples of which are given in Figure 4), and changes in the underlying physics of the model. The solar neutrino problem has been a major stimulus to models: in fact, most suggestions were motivated by the hope of producing a cooler sun $\left(T_{c} \sim 0.95 T_{c}\right)$ that would avoid conflict with the results of the ${ }^{37} \mathrm{Cl}$ experiment. The suggestions included models with low heavy element abundances ("low Z" models), in which one abandons the SSM assumption that the initial heavy element abundances are those we measure today at the sun's surface; periodically mixed solar cores; models where hydrogen is continually mixed into the core by turbulent diffusion or by convective mixing; and models where the solar core is partially supported by a strong central magnetic field or by its 


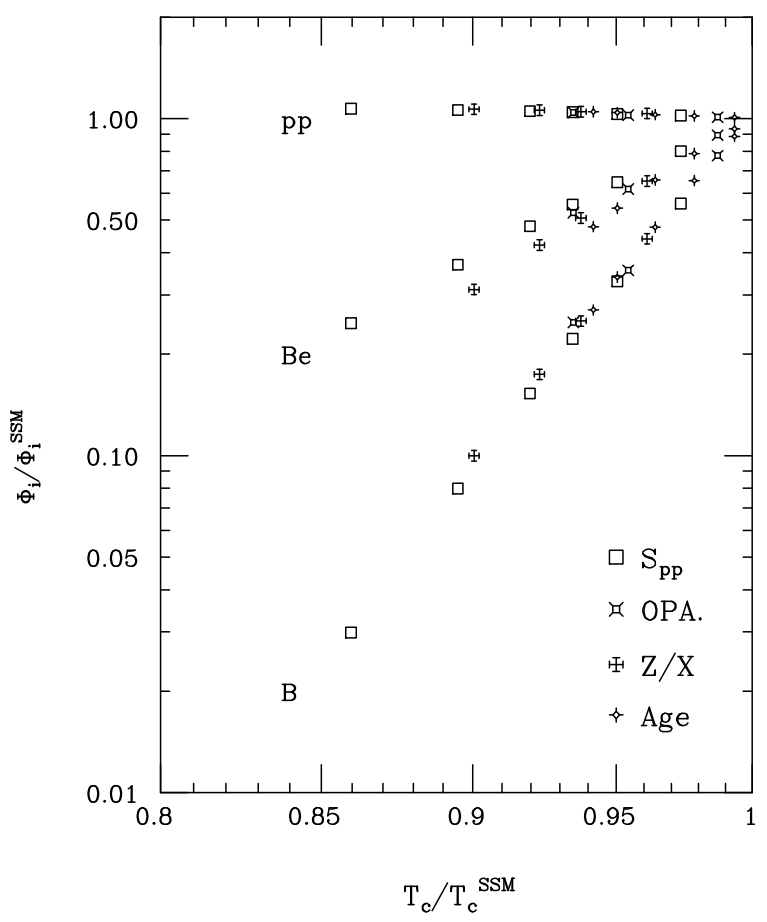

Figure 4: The responses of the pp, ${ }^{7} \mathrm{Be}$, and ${ }^{8} \mathrm{~B}$ neutrino fluxes to the indicated variations in solar model input parameters, displayed as a function of the resulting central temperature $T_{c}$. From Castellani et al.

rapid rotation, thereby relaxing the SSM assumption that hydrostatic equilibrium is achieved only through the gas pressure gradient. A larger list is given by Bahcall and Davis 19 . To illustrate the kinds of consequences such models have, two of these suggestions are discussed in more detail below.

In low- $\mathrm{Z}$ models one postulates a reduction in the core metallicity from $\mathrm{Z}$ $\sim 0.02$ to $\mathrm{Z} \sim 0.002$. This lowers the core opacity (primarily because metals are very important to free-bound electron transitions), thus reducing $T_{c}$ and weakening the ppII and ppIII cycles. The attractiveness of low- $\mathrm{Z}$ models is due in part to the existence of mechanisms for adding heavier elements to the sun's surface. These include the infall of comets and other debris, as well as the accumulation of dust as the sun passes through interstellar clouds. However, the increased radiative energy transport in low- $\mathrm{Z}$ models leads to a thin convective envelope, in contradiction to interpretations of the 5 -minute 
solar surface oscillations. A low He mass fraction also results. As diffusion of material from a thin convective envelope into the interior would deplete heavy elements at the surface, investigators have also questioned whether present abundances could have accumulated in low-Z models. Finally, the general consistency of solar heavy element abundances with those observed in other main sequence stars makes the model appear contrived.

Models in which the solar core $\left(\sim 0.2 \mathrm{M}_{\odot}\right)$ is intermittently mixed break the standard model assumption of a steady-state sun: for a period of several million years (the thermal relaxation time for the core) following mixing, the usual relationship between the observed surface luminosity and rate of energy (and neutrino) production is altered as the sun burns out of equilibrium. Calculations show that both the luminosity and the ${ }^{8} \mathrm{~B}$ neutrino flux are suppressed while the sun relaxes back to the steady state. Such models have been considered seriously because of instabilities associated with large gradients in the ${ }^{3} \mathrm{He}$ abundance, which in equilibrium varies as $\sim T^{-6}$, where $T$ is the local temperature. The resulting steep profile is unstable under finite amplitude displacements of a volume to smaller $\mathrm{r}$ : the energy released by the increased ${ }^{3} \mathrm{He}$ burning at higher $\mathrm{T}$ can exceed the energy in the perturbation. For a discussion of the plausibility of such a trigger for core mixing, one can see the original work of Dilke and Gough 20 as well as a more recent critique by Merryfield 21 1 . The possibility that continuous mixing on time scales of ${ }^{3} \mathrm{He}$ mixing could produce a flux pattern close to that observed (e.g., a suppression in both the ${ }^{8} \mathrm{~B}$ neutrino flux and the ${ }^{7} \mathrm{Be} /{ }^{8} \mathrm{~B}$ flux ratio) was recently discussed by Cumming and Haxton 22 .

This discussion of two of the more seriously explored nonstandard model possibilities illustrates how changes motivated by the solar neutrino problem often produce other, unwanted consequences. In particular, many experts feel that the good SSM agreement with helioseismology is likely to be destroyed by changes such as those discussed above.

Figure 5 is an illustration by Hata et al.23 of the flux predictions of several nonstandard models, including a low-Z model consistent with the ${ }^{37} \mathrm{Cl}$ results. As in the Castellani et al. exploration, the results cluster along a track that defines the naive $T_{c}$ dependence of the $\phi\left({ }^{7} \mathrm{Be}\right) / \phi\left({ }^{8} \mathrm{~B}\right)$ ratio, well separated from the experimental contours.

There is now a popular argument that no such nonstandard model can solve the solar neutrino problem: if one assumes undistorted neutrino spectra, no cembination of pp, ${ }^{7} \mathrm{Be}$, and ${ }^{8} \mathrm{~B}$ neutrino fluxes fits the experimental results well 24. In fact, in an unconstrained fit, the required ${ }^{7} \mathrm{Be}$ flux is unphysical, negative by almost $3 \sigma$. Thus, barring some unfortunate experimental error, it appears we are forced to look elsewhere for a solution. 


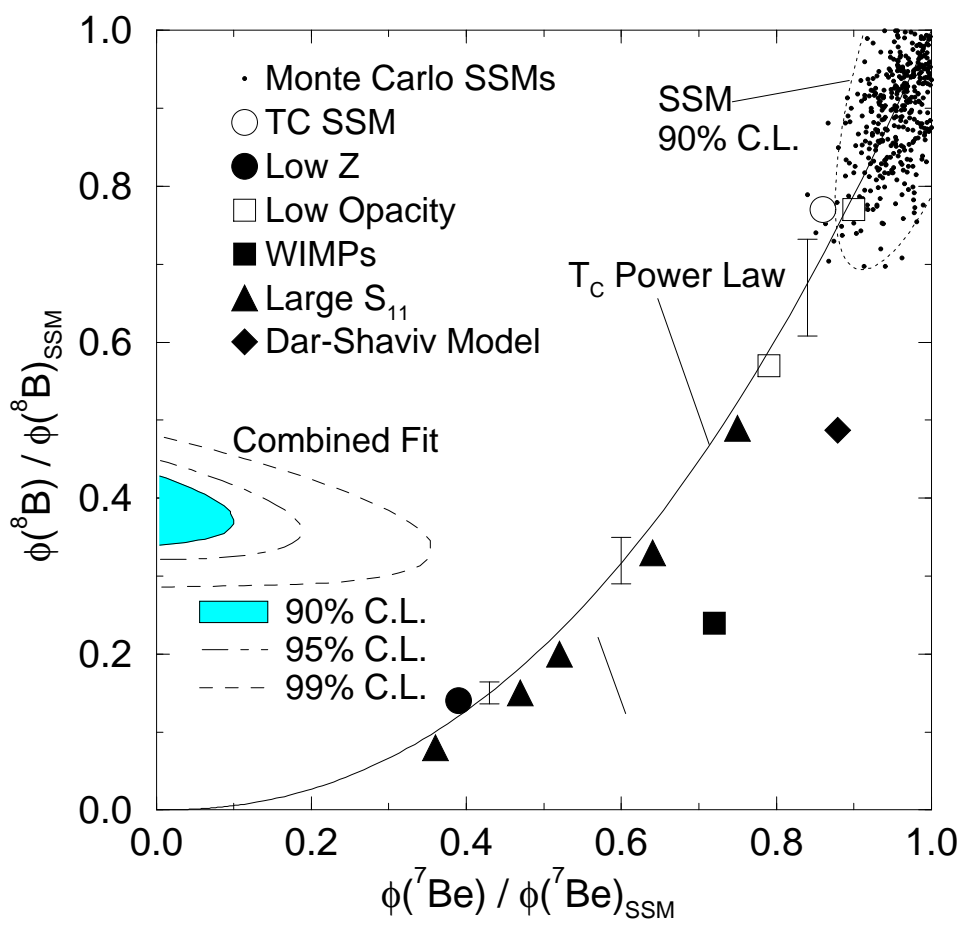

Figure 5: The fluxes allowed by the combined results of the various solar neutrino experiments compared to the results of SSM variations and various nonstandard solar models. The solid line in the naive $T_{c}$ power law discussed in the text. From Hata et al.

If experimental error, SSM parameter uncertainties, and nonstandard solar physics are ruled out as potential solutions, new particle physics is left as the leading possibility. Suggested particle physics solutions of the solar neutrino problem include neutrino oscillations, neutrino decay, neutrino magnetic moments, and weakly interacting massive particles. Among these, the MikheyevSmirnov-Wolfenstein effect - neutrino oscillations enhanced by matter interactions - is widely regarded as the most plausible.

\subsection{Neutrino Oscillations}

One odd feature of particle physics is that neutrinos, which are not required by any symmetry to be massless, nevertheless must be much lighter than any of the other known fermions. For instance, the current limit on the $\bar{\nu}_{e}$ mass is $\lesssim 5$ $\mathrm{eV}$. The standard model requires neutrinos to be massless, but the reasons are 
not fundamental. Dirac mass terms $m_{D}$, analogous to the mass terms for other fermions, cannot be constructed because the model contains no right-handed neutrino fields. Neutrinos can also have Majorana mass terms

$$
\overline{\nu_{L}^{c}} m_{L} \nu_{L} \quad \text { and } \quad \overline{\nu_{\mathrm{R}}^{c}} \mathrm{~m}_{\mathrm{R}} \nu_{\mathrm{R}}
$$

where the subscripts $L$ and $R$ denote left- and right-handed projections of the neutrino field $\nu$, and the superscript $c$ denotes charge conjugation. The first term above is constructed from left-handed fields, but can only arise as a nonrenormalizable effective interaction when one is constrained to generate $m_{L}$ with the doublet scalar field of the standard model. The second term is absent from the standard model because there are no right-handed neutrino fields.

None of these standard model arguments carries over to the more general, unified theories that theorists believe will supplant the standard model. In the enlarged multiplets of extended models it is natural to characterize the fermions of a single family, e.g., $\nu_{e}, \mathrm{e}, \mathrm{u}, \mathrm{d}$, by the same mass scale $m_{D}$. Small neutrino masses are then frequently explained as a result of the Majorana neutrino masses. In the seesaw mechanism,

$$
M_{\nu} \sim\left(\begin{array}{cc}
0 & m_{D} \\
m_{D}^{T} & m_{R}
\end{array}\right)
$$

Diagonalization of the mass matrix produces one light neutrino, $m_{\text {light }} \sim \frac{m_{D}^{2}}{m_{R}}$, and one unobservably heavy, $m_{\text {heavy }} \sim m_{R}$. The factor $\left(m_{D} / m_{R}\right)$ is the needed small parameter that accounts for the distinct scale of neutrino masses. The masses for the $\nu_{e}, \nu_{\mu}$, and $\nu_{\tau}$ are then related to the squares of the corresponding quark masses $m_{u}, m_{c}$, and $m_{t}$. Taking $m_{R} \sim 10^{16} \mathrm{GeV}$, a typical grand unification scale for models built on groups like $\mathrm{SO}(10)$, the seesaw mechanism gives the crude relation

$$
m_{\nu_{e}}: m_{\nu_{\mu}}: m_{\nu_{\tau}} \leftrightarrow 2 \cdot 10^{-12}: 2 \cdot 10^{-7}: 3 \cdot 10^{-3} \mathrm{eV}
$$

The fact that solar neutrino experiments can probe small neutrino masses, and thus provide insight into possible new mass scales $m_{R}$ that are far beyond the reach of direct accelerator measurements, has been an important theme of the field.

Now one of the most interesting possibilities for solving the solar neutrino problem has to do with neutrino masses. For simplicity we will discuss just two neutrinos. If a neutrino has a mass $m$, we mean that as it propagates through free space, its energy and momentum are related in the usual way for this mass. 
Thus if we have two neutrinos, we can label those neutrinos according to the eigenstates of the free Hamiltonian, that is, as mass eigenstates.

But neutrinos are produced by the weak interaction. In this case, we have another set of eigenstates, the flavor eigenstates. We can define a $\nu_{e}$ as the neutrino that accompanies the positron in $\beta$ decay. Likewise we label by $\nu_{\mu}$ the neutrino produced in muon decay.

Now the question: are the eigenstates of the free Hamiltonian and of the weak interaction Hamiltonian identical? Most likely the answer is no: we know this is the case with the quarks, since the different families (the analog of the mass eigenstates) do interact through the weak interaction. That is, the up quark decays not only to the down quark, but also occasionally to the strange quark. (This is why we had a $\cos \theta_{c}$ in our weak interaction amplitude: the amplitude for $u \rightarrow s$ is proportional to $\sin \theta_{c}$.) Thus we suspect that the weak interaction and mass eigenstates, while spanning the same two-neutrino space, are not coincident: the mass eigenstates $\left|\nu_{1}\right\rangle$ and $\left|\nu_{2}\right\rangle$ (with masses $m_{1}$ and $\left.m_{2}\right)$ are related to the weak interaction eigenstates by

$$
\begin{aligned}
& \left|\nu_{e}\right\rangle=\cos \theta_{v}\left|\nu_{1}\right\rangle+\sin \theta_{v}\left|\nu_{2}\right\rangle \\
& \left|\nu_{\mu}\right\rangle=-\sin \theta_{v}\left|\nu_{1}\right\rangle+\cos \theta_{v}\left|\nu_{2}\right\rangle
\end{aligned}
$$

where $\theta_{v}$ is the (vacuum) mixing angle.

An immediate consequence is that a state produced as a $\left|\nu_{e}\right\rangle$ or a $\left|\nu_{\mu}\right\rangle$ at some time $t$ - for example, a neutrino produced in $\beta$ decay — does not remain a pure flavor eigenstate as it propagates away from the source. This is because the different mass eigenstates comprising the neutrino will accumulate different phases as they propagate downstream, a phenomenon known as vacuum oscillations (vacuum because the experiment is done in free space). To see the effect, suppose we produce a neutrino in some $\beta$ decay where we measure the momentum of the initial nucleus, final nucleus, and positron. Thus the outgoing neutrino is a momentum eigenstate 25 . At time $t=0$

$$
|\nu(t=0)\rangle=\left|\nu_{e}\right\rangle=\cos \theta_{v}\left|\nu_{1}\right\rangle+\sin \theta_{v}\left|\nu_{2}\right\rangle
$$

Each eigenstate subsequently propagates with a phase

$$
e^{i(\vec{k} \cdot \vec{x}-\omega t)}=e^{i\left(\vec{k} \cdot \vec{x}-\sqrt{m_{i}^{2}+k^{2}} t\right)} .
$$

But if the neutrino mass is small compared to the neutrino momentum/energy, one can write

$$
\sqrt{m_{i}^{2}+k^{2}} \sim k\left(1+\frac{m_{i}^{2}}{2 k^{2}}\right) .
$$


Thus we conclude

$$
\begin{aligned}
|\nu(t)\rangle= & e^{i\left(\vec{k} \cdot \vec{x}-k t-\left(m_{1}^{2}+m_{2}^{2}\right) t / 4 k\right)} \\
& \times\left[\cos \theta_{v}\left|\nu_{1}\right\rangle e^{i \delta m^{2} t / 4 k}+\sin \theta_{v}\left|\nu_{2}\right\rangle e^{-i \delta m^{2} t / 4 k}\right] .
\end{aligned}
$$

We see there is a common average phase (which has no physical consequence) as well as a beat phase that depends on

$$
\delta m^{2}=m_{2}^{2}-m_{1}^{2} .
$$

Now it is a simple matter to calculate the probability that our neutrino state remains a $\left|\nu_{e}\right\rangle$ at time $\mathrm{t}$

$$
\begin{aligned}
P_{\nu_{e}}(t) & =\left|\left\langle\nu_{e} \mid \nu(t)\right\rangle\right|^{2} \\
& =1-\sin ^{2} 2 \theta_{v} \sin ^{2}\left(\frac{\delta m^{2} t}{4 k}\right) \rightarrow 1-\frac{1}{2} \sin ^{2} 2 \theta_{v}
\end{aligned}
$$

where the limit on the right is appropriate for large $t$. Now $E \sim k$, where $\mathrm{E}$ is the neutrino energy, by our assumption that the neutrino masses are small compared to $k$. Thus we can reinsert the units above to write the probability in terms of the distance $x$ of the neutrino from its source,

$$
P_{\nu}(x)=1-\sin ^{2} 2 \theta_{v} \sin ^{2}\left(\frac{\delta m^{2} c^{4} x}{4 \hbar c E}\right) .
$$

(When one properly describes the neutrino state as a wave packet, the largedistance behavior follows from the eventual separation of the mass eigenstates.) If the the oscillation length

$$
L_{o}=\frac{4 \pi \hbar c E}{\delta m^{2} c^{4}}
$$

is comparable to or shorter than one astronomical unit, a reduction in the solar $\nu_{e}$ flux would be expected in terrestrial neutrino oscillations.

The suggestion that the solar neutrino problem could be explained by neutrino oscillations was first made by Pontecorvo in 1958, who pointed out the analogy with $K_{0} \leftrightarrow \bar{K}_{0}$ oscillations. From the point of view of particle physics, the sun is a marvelous neutrino source. The neutrinos travel a long distance and have low energies $(\sim 1 \mathrm{MeV})$, implying a sensitivity to

$$
\delta m^{2} \gtrsim 10^{-12} e V^{2} .
$$

In the seesaw mechanism, $\delta m^{2} \sim m_{2}^{2}$, so neutrino masses as low as $m_{2} \sim$ $10^{-6} \mathrm{eV}$ could be probed. In contrast, terrestrial oscillation experiments with accelerator or reactor neutrinos are typically limited to $\delta m^{2} \gtrsim 0.1 \mathrm{eV}^{2}$. 
From the expressions above one expects vacuum oscillations to affect all neutrino species equally, if the oscillation length is small compared to an astronomical unit. This is somewhat in conflict with the data, as we have argued that the ${ }^{7} \mathrm{Be}$ neutrino flux is quite suppressed. Furthermore, there is a weak theoretical prejudice that $\theta_{v}$ should be small, like the Cabibbo angle. The first objection, however, can be circumvented in the case of "just so" oscillations where the oscillation length is comparable to one astronomical unit. In this case the oscillation probability becomes sharply energy dependent, and one can choose $\delta m^{2}$ to preferentially suppress one component (e.g., the monochromatic ${ }^{7}$ Be neutrinos). This scenario has been explored by several groups and remains an interesting possibility. However, the requirement of large mixing angles remains.

Below we will see that stars allow us to "get around" this problem with small mixing angles. In preparation for this, we first present the results above in a slightly more general way. The analog of eq. (24) for an initial muon neutrino $\left(|\nu(t=0)\rangle=\left|\nu_{\mu}\right\rangle\right)$ is

$$
\begin{aligned}
|\nu(t)\rangle= & e^{i\left(\vec{k} \cdot \vec{x}-k t-\left(m_{1}^{2}+m_{2}^{2}\right) t / 4 k\right)} \\
& \times\left[-\sin \theta_{v}\left|\nu_{1}\right\rangle e^{i \delta m^{2} t / 4 k}+\cos \theta_{v}\left|\nu_{2}\right\rangle e^{-i \delta m^{2} t / 4 k}\right]
\end{aligned}
$$

Now if we compare eqs. (24) and (30) we see that they are special cases of a more general problem. Suppose we write our initial neutrino wave function as

$$
|\nu(t=0)\rangle=a_{e}(t=0)\left|\nu_{e}\right\rangle+a_{\mu}(t=0)\left|\nu_{\mu}\right\rangle .
$$

Then eqs. (24) and (30) tell us that the subsequent propagation is described by changes in $a_{e}(x)$ and $a_{\mu}(x)$ according to (this takes a bit of algebra)

$$
i \frac{d}{d x}\left(\begin{array}{c}
a_{e} \\
a_{\mu}
\end{array}\right)=\frac{1}{4 E}\left(\begin{array}{cc}
-\delta m^{2} \cos 2 \theta_{v} & \delta m^{2} \sin 2 \theta_{v} \\
\delta m^{2} \sin 2 \theta_{v} & \delta m^{2} \cos 2 \theta_{v}
\end{array}\right)\left(\begin{array}{c}
a_{e} \\
a_{\mu}
\end{array}\right) .
$$

Note that the common phase has been ignored: it can be absorbed into the overall phase of the coeeficients $a_{e}$ and $a_{\mu}$, and thus has no consequence. Also, we have equated $x=t$, that is, set $c=1$.

\subsection{The Mikheyev-Smirnov-Wolfenstein Mechanism}

The view of neutrino oscillations changed when Mikheyev and Smirnov 26 showed in 1985 that the density dependence of the neutrino effective mass, a phenomenon first discussed by Wolfenstein in 1978, could greatly enhance oscillation probabilities: a $\nu_{e}$ is adiabatically transformed into a $\nu_{\mu}$ as it traverses a critical density within the sun. It became clear that the sun was not 
only an excellent neutrino source, but also a natural regenerator for cleverly enhancing the effects of flavor mixing.

While the original work of Mikheyev and Smirnov was numerical, their phenomenon was soon understood analytically as a level-crossing problem. If one writes the neutrino wave function in matter as in eq. (31), the evolution of $a_{e}(x)$ and $a_{\mu}(x)$ is governed by

$$
i \frac{d}{d x}\left(\begin{array}{c}
a_{e} \\
a_{\mu}
\end{array}\right)=\frac{1}{4 E}\left(\begin{array}{cr}
2 E \sqrt{2} G_{F} \rho(x)-\delta m^{2} \cos 2 \theta_{v} & \delta m^{2} \sin 2 \theta_{v} \\
\delta m^{2} \sin 2 \theta_{v} & -2 E \sqrt{2} G_{F} \rho(x)+\delta m^{2} \cos 2 \theta_{v}
\end{array}\right)\left(\begin{array}{c}
a_{e} \\
a_{\mu}
\end{array}\right)
$$

where $\mathrm{G}_{F}$ is the weak coupling constant and $\rho(x)$ the solar electron density. If $\rho(x)=0$, this is exactly our previous result and can be trivially integrated to give the vacuum oscillation solutions of Sec. 2.5. The new contribution to the diagonal elements, $2 E \sqrt{2} G_{F} \rho(x)$, represents the effective contribution to $M_{\nu}^{2}$ that arises from neutrino-electron scattering. The indices of refraction of electron and muon neutrinos differ because the former scatter by charged and neutral currents, while the latter have only neutral current interactions. The difference in the forward scattering amplitudes determines the densitydependent splitting of the diagonal elements of the new matter equation.

It is helpful to rewrite this equation in a basis consisting of the light and heavy local mass eigenstates (i.e., the states that diagonalize the right-hand side of the equation),

$$
\begin{aligned}
\left|\nu_{L}(x)\right\rangle & =\cos \theta(x)\left|\nu_{e}\right\rangle-\sin \theta(x)\left|\nu_{\mu}\right\rangle \\
\left|\nu_{H}(x)\right\rangle & =\sin \theta(x)\left|\nu_{e}\right\rangle+\cos \theta(x)\left|\nu_{\mu}\right\rangle .
\end{aligned}
$$

The local mixing angle is defined by

$$
\begin{aligned}
& \sin 2 \theta(x)=\frac{\sin 2 \theta_{v}}{\sqrt{X^{2}(x)+\sin ^{2} 2 \theta_{v}}} \\
& \cos 2 \theta(x)=\frac{-X(x)}{\sqrt{X^{2}(x)+\sin ^{2} 2 \theta_{v}}}
\end{aligned}
$$

where $X(x)=2 \sqrt{2} G_{F} \rho(x) E / \delta m^{2}-\cos 2 \theta_{v}$. Thus $\theta(x)$ ranges from $\theta_{v}$ to $\pi / 2$ as the density $\rho(x)$ goes from 0 to $\infty$.

If we define

$$
|\nu(x)\rangle=a_{H}(x)\left|\nu_{H}(x)\right\rangle+a_{L}(x)\left|\nu_{L}(x)\right\rangle,
$$

the neutrino propagation can be rewritten in terms of the local mass eigenstates

$$
i \frac{d}{d x}\left(\begin{array}{c}
a_{H} \\
a_{L}
\end{array}\right)=\left(\begin{array}{cc}
\lambda(x) & i \alpha(x) \\
-i \alpha(x) & -\lambda(x)
\end{array}\right)\left(\begin{array}{c}
a_{H} \\
a_{L}
\end{array}\right)
$$


with the splitting of the local mass eigenstates determined by

$$
2 \lambda(x)=\frac{\delta m^{2}}{2 E} \sqrt{X^{2}(x)+\sin ^{2} 2 \theta v}
$$

and with mixing of these eigenstates governed by the density gradient

$$
\alpha(x)=\left(\frac{E}{\delta m^{2}}\right) \frac{\sqrt{2} G_{F} \frac{d}{d x} \rho(x) \sin 2 \theta_{v}}{X^{2}(x)+\sin ^{2} 2 \theta_{v}} .
$$

The results above are quite interesting: the local mass eigenstates diagonalize the matrix if the density is constant. In such a limit, the problem is no more complicated than our original vacuum oscillation case, although our mixing angle is changed because of the matter effects. But if the density is not constant, the mass eigenstates in fact evolve as the density changes. This is the crux of the MSW effect. Note that the splitting achieves its minimum value, $\frac{\delta m^{2}}{2 E} \sin 2 \theta_{v}$, at a critical density $\rho_{c}=\rho\left(x_{c}\right)$

$$
2 \sqrt{2} E G_{F} \rho_{c}=\delta m^{2} \cos 2 \theta_{v}
$$

that defines the point where the diagonal elements of the original flavor matrix cross.

Our local-mass-eigenstate form of the propagation equation can be trivially integrated if the splitting of the diagonal elements is large compared to the offdiagonal elements,

$$
\gamma(x)=\left|\frac{\lambda(x)}{\alpha(x)}\right|=\frac{\sin ^{2} 2 \theta_{v}}{\cos 2 \theta_{v}} \frac{\delta m^{2}}{2 E} \frac{1}{\left|\frac{1}{\rho_{c}} \frac{d \rho(x)}{d x}\right|} \frac{\left[X(x)^{2}+\sin ^{2} 2 \theta_{v}\right]^{3 / 2}}{\sin ^{3} 2 \theta_{v}} \gg 1,
$$

a condition that becomes particularly stringent near the crossing point,

$$
\gamma_{c}=\gamma\left(x_{c}\right)=\frac{\sin ^{2} 2 \theta_{v}}{\cos 2 \theta_{v}} \frac{\delta m^{2}}{2 E} \frac{1}{\left|\frac{1}{\rho_{c}} \frac{d \rho(x)}{d x}\right|_{x=x_{c}} \mid} \gg 1 .
$$

The resulting adiabatic electron neutrino survival probability 27, valid when $\gamma_{c} \gg 1$, is

$$
P_{\nu_{e}}^{\text {adiab }}=\frac{1}{2}+\frac{1}{2} \cos 2 \theta_{v} \cos 2 \theta_{i}
$$

where $\theta_{i}=\theta\left(x_{i}\right)$ is the local mixing angle at the density where the neutrino was produced.

The physical picture behind this derivation is illustrated in Fig. 6. One makes the usual assumption that, in vacuum, the $\nu_{e}$ is almost identical to the 


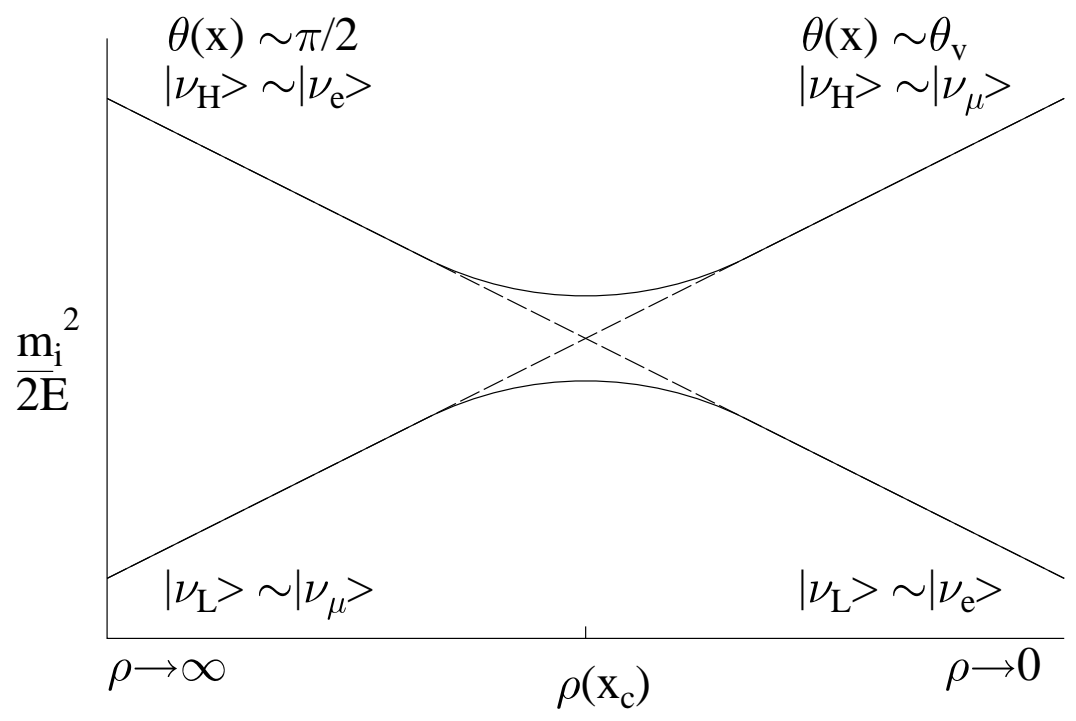

Figure 6: Schematic illustration of the MSW crossing. The dashed lines correspond to the electron-electron and muon-muon diagonal elements of the $M^{2}$ matrix in the flavor basis. Their intersection defines the level-crossing density $\rho_{c}$. The solid lines are the trajectories of the light and heavy local mass eigenstates. If the electron neutrino is produced at high density and propagates adiabatically, it will follow the heavy-mass trajectory, emerging from the sun as a $\nu_{\mu}$.

light mass eigenstate, $\nu_{L}(0)$, i.e., $m_{1}<m_{2}$ and $\cos \theta_{v} \sim 1$. But as the density increases, the matter effects make the $\nu_{e}$ heavier than the $\nu_{\mu}$, with $\nu_{e} \rightarrow \nu_{H}(x)$ as $\rho(x)$ becomes large. The special property of the sun is that it produces $\nu_{e} \mathrm{~S}$ at high density that then propagate to the vacuum where they are measured. The adiabatic approximation tells us that if initially $\nu_{e} \sim \nu_{H}(x)$, the neutrino will remain on the heavy mass trajectory provided the density changes slowly. That is, if the solar density gradient is sufficiently gentle, the neutrino will emerge from the sun as the heavy vacuum eigenstate, $\sim \nu_{\mu}$. This guarantees nearly complete conversion of $\nu_{e} \mathrm{~s}$ into $\nu_{\mu} \mathrm{s}$, producing a flux that cannot be detected by the Homestake or SAGE/GALLEX detectors.

But this does not explain the curious pattern of partial flux suppressions coming from the various solar neutrino experiments. The key to this is the behavior when $\gamma_{c} \lesssim 1$. Our expression for $\gamma(x)$ shows that the critical region for nonadiabatic behavior occurs in a narrow region (for small $\theta_{v}$ ) surrounding the crossing point, and that this behavior is controlled by the derivative of the 
density. This suggests an analytic strategy for handling nonadiabatic crossings: one can replace the true solar density by a simpler (integrable!) two-parameter form that is constrained to reproduce the true density and its derivative at the crossing point $x_{c}$. Two convenient choices are the linear $(\rho(x)=a+$ $b x)$ and exponential $\left(\rho(x)=a e^{-b x}\right)$ profiles. As the density derivative at $x_{c}$ governs the nonadiabatic behavior, this procedure should provide an accurate description of the hopping probability between the local mass eigenstates when the neutrino traverses the crossing point. The initial and ending points $x_{i}$ and $x_{f}$ for the artificial profile are then chosen so that $\rho\left(x_{i}\right)$ is the density where the neutrino was produced in the solar core and $\rho\left(x_{f}\right)=0$ (the solar surface), as illustrated in in Fig. 7 . Since the adiabatic result $\left(P_{\nu_{e}}^{\text {adiab }}\right)$ depends only on the local mixing angles at these points, this choice builds in that limit. But our original flavor-basis equation can then be integrated exactly for linear and exponential profiles, with the results given in terms of parabolic cylinder and Whittaker functions, respectively.

That result can be simplified further by observing that the nonadiabatic region is generally confined to a narrow region around $x_{c}$, away from the endpoints $x_{i}$ and $x_{f}$. We can then extend the artificial profile to $x= \pm \infty$, as illustrated by the dashed lines in Fig. 7. As the neutrino propagates adiabatically in the unphysical region $x<x_{i}$, the exact soluation in the physical region can be recovered by choosing the initial boundary conditions

$$
\begin{aligned}
& a_{L}(-\infty)=-a_{\mu}(-\infty)=\cos \theta_{i} e^{-i \int_{-\infty}^{x_{i}} \lambda(x) d x} \\
& a_{H}(-\infty)=a_{e}(-\infty)=\sin \theta_{i} e^{i \int_{-\infty}^{x_{i}} \lambda(x) d x} .
\end{aligned}
$$

That is, $|\nu(-\infty)\rangle$ will then adiabatically evolve to $\left|\nu\left(x_{i}\right)\right\rangle=\left|\nu_{e}\right\rangle$ as $x$ goes from $-\infty$ to $x_{i}$. The unphysical region $x>x_{f}$ can be handled similarly.

With some algebra a simple generalization of the adiabatic result emerges that is valid for all $\delta m^{2} / E$ and $\theta_{v}$

$$
P_{\nu_{e}}=\frac{1}{2}+\frac{1}{2} \cos 2 \theta_{v} \cos 2 \theta_{i}\left(1-2 P_{\mathrm{hop}}\right)
$$

where $\mathrm{P}_{\text {hop }}$ is the Landau-Zener probability of hopping from the heavy mass trajectory to the light trajectory on traversing the crossing point. For the linear approximation to the density 22 , es,

$$
P_{\text {hop }}^{\operatorname{lin}}=e^{-\pi \gamma_{c} / 2} .
$$

As it must by our construction, $P_{\nu_{e}}$ reduces to $\mathrm{P}_{\nu_{e}}^{\text {adiab }}$ for $\gamma_{c} \gg 1$. When the crossing becomes nonadiabatic (e.g., $\gamma_{c} \ll 1$ ), the hopping probability goes to 


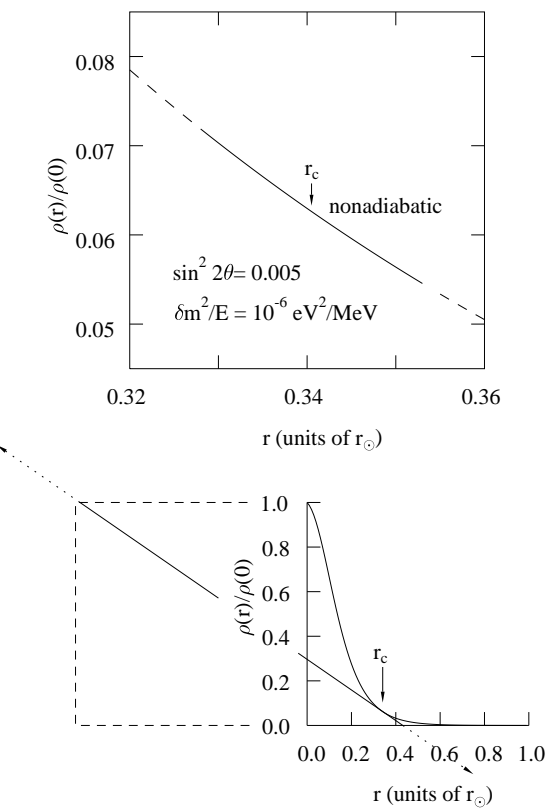

Figure 7: The top figure illustrates, for one choice of $\sin ^{2} 2 \theta$ and $\delta m^{2}$, that the region of nonadiabatic propagation (solid line) is usually confined to a narrow region around the crossing point $r_{c}$. In the lower figure, the solid lines represent the solar density and a linear approximation to that density that has the correct initial and final values, as well as the correct density and density derivative at $r_{c}$. Thus the linear profile is a very good approximation to the sun in the vicinity of the crossing point. The MSW equations can be solved analytically for this wedge. By extending the wedge to $\pm \infty$ (dotted lines) and assuming adiabatic propagation in these regions of unphysical density, one obtains the simple Landau-Zener result discussed in the text.

1, allowing the neutrino to exit the sun on the light mass trajectory as a $\nu_{e}$, i.e., no conversion occurs.

Thus there are two conditions for strong conversion of solar neutrinos: there must be a level crossing (that is, the solar core density must be sufficient to render $\nu_{e} \sim \nu_{H}\left(x_{i}\right)$ when it is first produced) and the crossing must be adiabatic. The first condition requires that $\delta m^{2} / E$ not be too large, and the second $\gamma_{c} \gtrsim 1$. The combination of these two constraints, illustrated in Fig. 8 , defines a triangle of interesting parameters in the $\frac{\delta m^{2}}{E}-\sin ^{2} 2 \theta_{v}$ plane, as Mikheyev and Smirnov found by numerically integration. A remarkable feature of this triangle is that strong $\nu_{e} \rightarrow \nu_{\mu}$ conversion can occur for very 
small mixing angles $\left(\sin ^{2} 2 \theta \sim 10^{-3}\right)$, unlike the vacuum case.

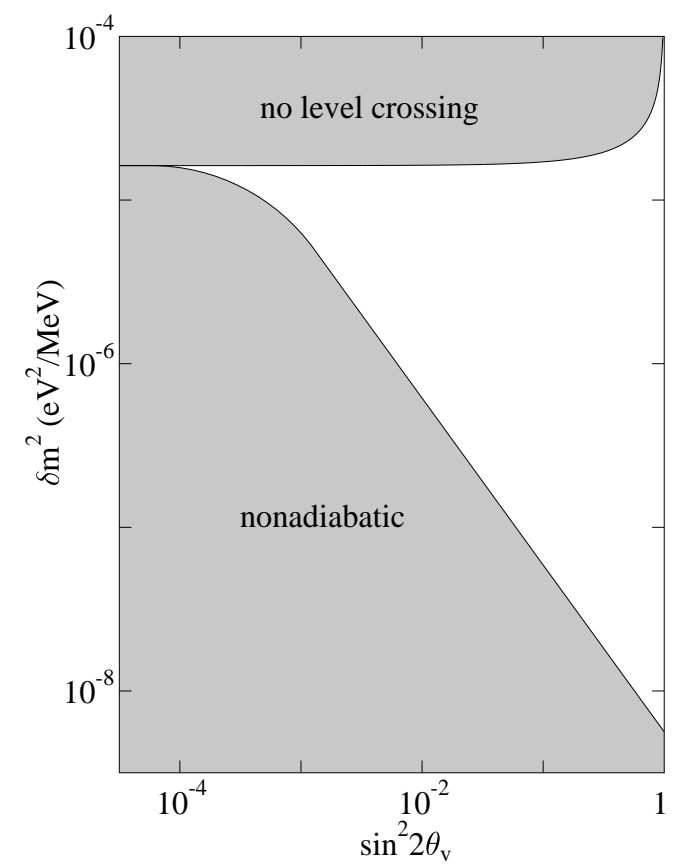

Figure 8: MSW conversion for a neutrino produced at the sun's center. The upper shaded region indices thoses $\delta m^{2} / E$ where the vacuum mass splitting is too great to be overcome by the solar density. Thus no level crossing occurs. The lower shaded region defines the region where the level crossing is nonadiabatic ( $\gamma_{c}$ less than unity). The unshaded region corresponds to adiabatic level crossings where strong $\nu_{e} \rightarrow \nu_{\mu}$ will occur.

One can envision superimposing on Fig. 8 the spectrum of solar neutrinos, plotted as a function of $\frac{\delta m^{2}}{E}$ for some choice of $\delta m^{2}$. Since Davis sees some solar neutrinos, the solutions must correspond to the boundaries of the triangle in Fig. 8. The horizontal boundary indicates the maximum $\frac{\delta m^{2}}{E}$ for which the sun's central density is sufficient to cause a level crossing. If a spectrum properly straddles this boundary, we obtain a result consistent with the Homestake experiment in which low energy neutrinos (large 1/E) lie above the level-crossing boundary (and thus remain $\nu_{e}$ 's), but the high-energy neutrinos (small 1/E) fall within the unshaded region where strong conversion takes place. Thus such a solution would mimic nonstandard solar models in that only the ${ }^{8} \mathrm{~B}$ neutrino flux would be strongly suppressed. The diagonal 
boundary separates the adiabatic and nonadiabatic regions. If the spectrum straddles this boundary, we obtain a second solution in which low energy neutrinos lie within the conversion region, but the high-energy neutrinos (small $1 / \mathrm{E})$ lie below the conversion region and are characterized by $\gamma \ll 1$ at the crossing density. (Of course, the boundary is not a sharp one, but is characterized by the Landau-Zener exponential). Such a nonadiabatic solution is quite distinctive since the flux of pp neutrinos, which is strongly constrained in the standard solar model and in any steady-state nonstandard model by the solar luminosity, would now be sharply reduced. Finally, one can imagine "hybrid" solutions where the spectrum straddles both the level-crossing (horizontal) boundary and the adiabaticity (diagonal) boundary for small $\theta$, thereby reducing the ${ }^{7} \mathrm{Be}$ neutrino flux more than either the pp or ${ }^{8} \mathrm{~B}$ fluxes.

What are the results of a careful search for MSW solutions satisfying the Homestake, Kamiokande/SuperKamiokande, and SAGE/GALLEX constraints? This has been done by several groups: recent results will be discussed by N. Hata in his lectures. One solution, corresponding to a region surrounding $\delta m^{2} \sim 6 \cdot 10^{-6} \mathrm{eV}^{2}$ and $\sin ^{2} 2 \theta_{v} \sim 6 \cdot 10^{-3}$, is the hybrid case described above. It is commonly called the small-angle solution. A second, large-angle solution exists, corresponding to $\delta m^{2} \sim 10^{-5} \mathrm{eV}^{2}$ and $\sin ^{2} 2 \theta_{v} \sim 0.6$. These solutions can be distinguished by their characteristic distortions of the solar neutrino spectrum. The survival probabilities $P_{\nu_{e}}^{\mathrm{MSW}}(\mathrm{E})$ for the small- and large-angle parameters given above are shown as a function of $\mathrm{E}$ in Fig. 9.

The MSW mechanism provides a natural explanation for the pattern of observed solar neutrino fluxes. While it requires profound new physics, both massive neutrinos and neutrino mixing are expected in extended models. The small-angle solution corresponds to $\delta m^{2} \sim 10^{-5} \mathrm{eV}^{2}$, and thus is consistent with $m_{2} \sim$ few $\cdot 10^{-3} \mathrm{eV}$. This is a typical $\nu_{\tau}$ mass in models where $m_{R} \sim$ $m_{\mathrm{GUT}}$. This mass is also reasonably close to atmospheric neutrino values. On the other hand, if it is the $\nu_{\mu}$ participating in the oscillation, this gives $m_{R} \sim 10^{12} \mathrm{GeV}$ and predicts a heavy $\nu_{\tau} \sim 10 \mathrm{eV}$. Such a mass is of great interest cosmologically as it would have consequences for supernova physics, the dark matter problem, and the formation of large-scale structure.

\subsection{Spin-Flavor Oscillations}

If the MSW mechanism proves not to be the solution of the solar neutrino problem, it still will have greatly enhanced the importance of solar neutrino physics: the existing experiments have ruled out large regions in the $\delta m^{2}-\sin ^{2} 2 \theta_{v}$ plane (corresponding to nearly complete $\nu_{e} \rightarrow \nu_{\mu}$ conversion) that remain hopelessly beyond the reach of accelerator neutrino oscillation experiments. 


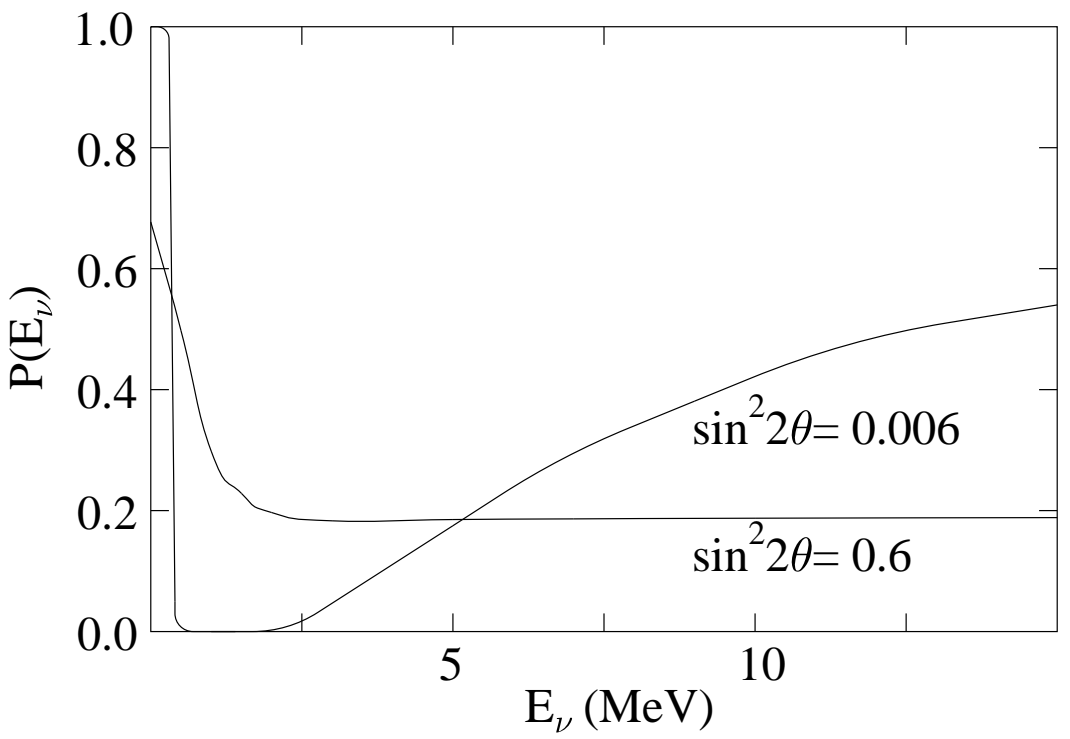

Figure 9: MSW survival probabilities $\mathrm{P}\left(\mathrm{E}_{\nu}\right)$ for typical small angle and large angle solutions.

A number of other particle physics solutions have been considered, such as neutrino decay, matter-catalyzed neutrino decay, and solar energy transport by weakly interacting massive particles. But perhaps the most interesting possibility, apart from the MSW mechanism, was stimulated by suggestions that the ${ }^{37} \mathrm{Cl}$ signal might be varying with a period comparable to the 11year solar cycle. While the evidence for this has weakened, the original claims generated renewed interest in neutrino magnetic moment interactions with the solar magnetic field.

The original suggestions by Cisneros and by Okun, Voloshyn, and Vysotsky envisioned the rotation

$$
\nu_{e_{L}} \rightarrow \nu_{e_{R}}
$$

producing a right-handed neutrino with sterile interactions in the standard model. With the discovery of the MSW mechanism, it was realized that matter effects would break the vacuum degeperacy of the $\nu_{e_{L}}$ and $\nu_{e_{R}}$, suppressing the spin precession. Lim and Marciano 30 and Akmedov 31 pointed out that this difficulty was naturally circumvented for

$$
\nu_{e_{L}} \rightarrow \nu_{\mu_{R}}
$$


as the different matter interactions of the $\nu_{e}$ and $\nu_{\mu}$ can compensate for the vacuum $\nu_{e}-\nu_{\mu}$ mass difference, producing a crossing similar to the usual MSW mechanism. Such spin-flavor precession can then occur at full strength due to an off-diagonal (in flavor) magnetic moment.

Quite relevant to this suggestion is the very strong limit on both diagonal and off-diagonal magnetic moments is imposed by studies of the red giant cooling process of plasmon decay into neutrinos

$$
\gamma^{*} \rightarrow \nu_{i} \bar{\nu}_{j}
$$

The result is $\left|\mu_{i j}\right| \lesssim 3 \cdot 10^{-12} \mu_{B}$, where $\mu_{B}$ is an electron Bohr magneton 32 . (This can be compared to a simple one-loop estimate 33 of the neutrino magnetic moment of $\sim 10^{-18} \mu_{B}$, taking a typical dark matter value for the neutrino mass of a few eV.) If a magnetic moment at the red giant limit is assumed, it follows that solar magnetic field strengths of $B_{\odot} \gtrsim 10^{6} G$ are needed to produce interesting effects. Since the location of the spin-flavor level crossing depends on the neutrino energy, such fields have to be extensive to affect an appreciable fraction of the neutrino spectrum. It is unclear whether these conditions can occur in the sun. This constraint from stellar cooling leads us naturally into our next lecture.

\section{Dirac and Majorana Neutrinos and Stellar Cooling}

\subsection{The Neutrino Mass Matrix}

Consider a general $4 \mathrm{n} \times 4 \mathrm{n}$ neutrino mass matrix where $\mathrm{n}$ is the number of flavors

$$
\left(\bar{\Psi}_{L}^{c}, \bar{\Psi}^{R}, \bar{\Psi}_{L}, \bar{\Psi}_{R}^{c}\right)\left(\begin{array}{cccc}
0 & 0 & M_{L} & M_{D}^{T} \\
0 & 0 & M_{D} & M_{R}^{\dagger} \\
M_{L}^{\dagger} & M_{D}^{\dagger} & 0 & 0 \\
M_{D}^{*} & M_{R} & 0 & 0
\end{array}\right)\left(\begin{array}{c}
\Psi_{L}^{c} \\
\Psi_{R} \\
\Psi_{L} \\
\Psi_{R}^{c}
\end{array}\right)
$$

where each entry in this matrix is understood to be a $\mathrm{n} \times \mathrm{n}$ matrix operating in flavor space. The entries $M_{D}$ are the Dirac mass terms, while the $M_{L}$ and $M_{R}$ are the Majorana terms. The latter break the invariance of the Dirac equation under the transformation $\psi(x) \rightarrow e^{i \alpha} \psi(x)$ associated with a conserved lepton number. Thus it is these terms that govern lepton-number-violating processes like double beta decay.

One can proceed to diagonalize this matrix

$$
\Psi_{\nu(e)}^{L}=\sum_{i=1}^{2 n} U_{e i}^{L} \tilde{\nu}_{i}(x) \quad \text { with masses } \mathrm{m}_{\mathrm{i}} .
$$


The eigenstates are two-component Majorana neutrinos 34 , yielding the proper $2 \times 2 n=4 n$ degrees of freedom, where $n$ is the number of flavors. We can recover the Majorana and Dirac limits:

- If $M_{R}=M_{L}=0$, the eigenstates of this matrix become pairwise degenerate, allowing the $2 n$ two-component eigenstates to be paired to form $n$ fourcomponent Dirac eigenstates.

- If $M_{D}=0$, the left- and right-handed components decouple, yielding $n$ lefthanded Majorana eigenstates with standard model interactions.

There are interesting physical effects associated with these limits. Dirac neutrinos can have magnetic dipole, electric dipole (CP and $\mathrm{T}$ violating), and anapole ( $\mathrm{P}$ violating) moments, as well as nonzero charge radii. Majorana neutrinos can have anapole moments but only transition magnetic and electric dipole moments. I mentioned in the previous lecture that transition magnetic moments were quite interesting in the context of MSW effects, as well as the use of $M_{R}$ in the seesaw mechanism.

\subsection{Red Giants and Helium Burning}

We now consider the evolution off the main sequence of a solar-like star, with a mass above half a solar mass. As the hydrogen burning in the core progresses to the point that no more hydrogen is available, the stellar core consists of the ashes from this burning, ${ }^{4} \mathrm{He}$. The star then goes through an interesting evolution:

- With no further means of producing energy, the core slowly contracts, thereby increasing in temperature as gravity does work on the core.

- Matter outside the core is still hydrogen rich, and can generate energy through hydrogen burning. Thus burning in this shell of material supports the outside layers of the star. Note as the core contracts, this matter outside the core also is pulled deeper into the gravitational potential. Furthermore, the shell $\mathrm{H}$ burning continually adds more mass to the core. This means the burning in the shell must intensify to generate the additional gas pressure to fight gravity. The shell also thickens as this happens, since more hydrogen is above the burning temperature.

- The resulting increasing gas pressure causes the outer envelope of the star to expand by a larger factor, up to a factor of 50 . The increase in radius more than compensates for the increased internal energy generation, so that a cooler surface results. The star reddens. Thus this class of star is named a red supergiant.

- This evolution is relatively rapid, perhaps a few hundred million years: the dense core requires large energy production. The helium core is supported by 
its degeneracy pressure, and is characterized by densities $\sim 10^{6} \mathrm{~g} / \mathrm{cm}^{3}$. This stage ends when the core reaches densities and temperatures that allow helium burning through the reaction

$$
\alpha+\alpha+\alpha \rightarrow^{12} C+\gamma
$$

As this reaction is very temperature dependent (see below), the conditions for ignition are very sharply defined. This has the consequence that the core mass at the helium flash point is well determined.

- The onset of helium burning produces a new source of support for the core. The energy release elevates the temperature and the core expands: He burning, not electron degeneracy, now supports the core. The burning shell and envelope have moved outward, higher in the gravitational potential. Thus shell hydrogen burning slows (the shell cools) because less gas pressure is needed to satisfy hydrostatic equilibrium. All of this means the evolution of the star has now slowed: the red giant moves along the "horizontal branch", as interior temperatures slowly elevate much as in the main sequence.

The $3 \alpha$ process depends on some rather interesting nuclear physics. The first interesting "accident" involves the near degeneracy of the ${ }^{8} \mathrm{Be}$ ground state and two separated $\alpha$ s: The ${ }^{8} \mathrm{Be} 0^{+}$ground state is just $92 \mathrm{keV}$ above the $2 \alpha$ threshold. The measured width of the ${ }^{8} \mathrm{Be}$ ground state is $2.5 \mathrm{eV}$, which corresponds to a lifetime of

$$
\tau_{m} \sim 2.6 \cdot 10^{-16} \mathrm{~s} .
$$

One can compare this number to the typical time for one $\alpha$ to pass another. The red giant core temperature is $T_{7} \sim 10 \rightarrow E \sim 8.6 \mathrm{keV}$. Thus v/c $\sim 0.002$. So the transit time is

$$
\tau \sim \frac{d}{v} \sim \frac{5 f}{0.002} \frac{1}{3 \cdot 10^{10} \mathrm{~cm} / \mathrm{sec}} \frac{10^{-13} \mathrm{~cm}}{\mathrm{f}} \sim 8 \cdot 10^{-21} \mathrm{~s} .
$$

This is more than five orders of magnitude shorter than $\tau_{m}$ above. Thus when a ${ }^{8} \mathrm{Be}$ nucleus is produced, it lives for a substantial time compared to this naive estimate.

To quantify this, we calculate the flux-averaged cross section assuming resonant capture

$$
\langle\sigma v\rangle=\left(\frac{2 \pi}{\mu k T}\right)^{3 / 2} \frac{\Gamma \Gamma}{\Gamma} e^{-E_{r} / k T}
$$

where $\Gamma$ is the $2 \alpha$ width of the ${ }^{8} \mathrm{Be}$ ground state. This is the cross section for the $\alpha+\alpha$ reaction to form the compound nucleus then decay by $\alpha+\alpha$. But 
since there is only one channel, this is clearly also the result for producing the compound nucleus ${ }^{8} \mathrm{Be}$.

By multiplying the rate/volume for producing ${ }^{8} \mathrm{Be}$ by the lifetime of ${ }^{8} \mathrm{Be}$, one gets the number of ${ }^{8} \mathrm{Be}$ nuclei per unit volume

$$
\begin{aligned}
N(B e) & =\frac{N_{\alpha} N_{\alpha}}{1+\delta_{\alpha \alpha}}\langle\sigma v\rangle \tau_{m} \\
& =\frac{N_{\alpha} N_{\alpha}}{1+\delta_{\alpha \alpha}}\langle\sigma v\rangle \frac{1}{\Gamma} \\
& =\frac{N_{\alpha}^{2}}{2}\left(\frac{2 \pi}{\mu k T}\right)^{3 / 2} e^{-E_{r} / k T} .
\end{aligned}
$$

Notice that the concentration is independent of $\Gamma$. So a small $\Gamma$ is not the reason we obtain a substantial buildup of ${ }^{8} \mathrm{Be}$. This is easily seen: if the width is small, then the production rate of ${ }^{8} \mathrm{Be}$ goes down, but the lifetime of the nucleus once it is produced is longer. The two effects cancel to give the same ${ }^{8} \mathrm{Be}$ concentration. One sees that the significant ${ }^{8} \mathrm{Be}$ concentration results from two effects: 1) $\alpha+\alpha$ is the only open channel and 2) the resonance energy is low enough that some small fraction of the $\alpha+\alpha$ reactions have the requisite energy. As $E_{r}=92 \mathrm{keV}, E_{r} / k T=10.67 / T_{8}$ (where $T_{8}$ is the temperature in $10^{8} \mathrm{~K}$ ) so that

$$
N(B e)=N_{\alpha}^{2} T_{8}^{-3 / 2} e^{-10.67 / T_{8}}\left(0.94 \cdot 10^{-33} \mathrm{~cm}^{3}\right) .
$$

So plugging in typical values of $N_{\alpha} \sim 1.5 \cdot 10^{28} / \mathrm{cm}^{3}$ (corresponding to $\rho_{\alpha} \sim 10^{5}$ $\left.\mathrm{g} / \mathrm{cm}^{3}\right)$ and $T_{8} \sim 1$ yields

$$
\frac{N\left({ }^{8} B e\right)}{N(\alpha)} \sim 3.2 \times 10^{-10}
$$

Salpeter suggested that this concentration would then allow $\alpha+{ }^{8} \mathrm{Be} \rightarrow{ }^{12} \mathrm{C}$ to take place. Hoyle then argued that this reaction would not be fast enough to produce significant burning unless it was also resonant. Now the mass of ${ }^{8} \mathrm{Be}+\alpha$, relative to ${ }^{12} \mathrm{C}$, is $7.366 \mathrm{MeV}$, and each nucleus has $J^{\pi}=0^{+}$. Thus s-wave capture would require a $0^{+}$resonance in ${ }^{12} \mathrm{C}$ at $\sim 7.4 \mathrm{MeV}$. No such state was then known, but a search by Cook, Fowler, Lauritsen, and Lauritsen revealed a $0^{+}$level at $7.644 \mathrm{MeV}$, with decay channels ${ }^{8} \mathrm{Be}+\alpha$ and $\gamma$ decay to the $2^{+} 4.433$ level in ${ }^{12} \mathrm{C}$. The parameters are

$$
\Gamma_{\alpha} \sim 8.9 \mathrm{eV} \quad \Gamma_{\gamma} \sim 3.6 \cdot 10^{-3} \mathrm{eV} .
$$

The resonant cross section formula gives

$$
r_{48}=N_{8} N_{\alpha}\left(\frac{2 \pi}{\mu k T}\right)^{3 / 2} \frac{\Gamma_{\alpha} \Gamma_{\gamma}}{\Gamma} e^{-E_{r} / k T} .
$$


Plugging in our previous expression for $N\left({ }^{8} \mathrm{Be}\right)$ yields

$$
r_{48}=N_{\alpha}^{3} T_{8}^{-3} e^{-42.9 / T_{8}}\left(6.3 \cdot 10^{-54} \mathrm{~cm}^{6} / \mathrm{s}\right) .
$$

If we denote by $\omega_{3 \alpha}$ the decay rate of an $\alpha$ in our plasma, then

$$
\begin{aligned}
\omega_{3 \alpha} & =3 N_{\alpha}^{2} T_{8}^{-3} e^{-42.9 / T_{8}}\left(6.3 \cdot 10^{-54} \mathrm{~cm}^{6} / \mathrm{sec}\right) \\
& =\left(\frac{N_{\alpha}}{1.5 \cdot 10^{28} / \mathrm{cm}^{3}}\right)^{2}\left(4.3 \cdot 10^{3} / \mathrm{sec}\right) \mathrm{T}_{8}^{-3} \mathrm{e}^{-42.9 / \mathrm{T}_{8}} .
\end{aligned}
$$

Now the energy release per reaction is $7.27 \mathrm{MeV}$. Thus we can calculate the energy produced per gram, $\epsilon$ :

$$
\begin{aligned}
\epsilon & =\omega_{3 \alpha} \frac{7.27 \mathrm{MeV}}{3} \frac{1.5 \cdot 10^{23}}{\mathrm{~g}} \\
& =\left(2.5 \cdot 10^{21} \mathrm{erg} / \mathrm{g} \mathrm{sec}\right)\left(\frac{\mathrm{N}_{\alpha}}{1.5 \cdot 10^{28} / \mathrm{cm}^{3}}\right)^{2} \mathrm{~T}_{8}^{-3} \mathrm{e}^{-42.9 / \mathrm{T}_{8}} .
\end{aligned}
$$

We can evaluate this at a temperature of $T_{8} \sim 1$ to find

$$
\epsilon \sim(584 \mathrm{ergs} / \mathrm{g} \mathrm{sec})\left(\frac{\mathrm{N}_{\alpha}}{1.5 \cdot 10^{28} / \mathrm{cm}^{3}}\right)^{2} .
$$

Typical values found in stellar calculations are in good agreement with this: typical red giant energy production is $\sim 100$ ergs per gram per second.

To get a feel for the temperature sensitivity of this process, we can do a Taylor series expansion, finding

$$
\epsilon(T) \sim\left(\frac{T}{T_{o}}\right)^{40} N_{\alpha}^{2}
$$

This steep temperature dependence is the reason the He flash is delicately dependent on conditions in the core.

\subsection{Neutrino Magnetic Moments and He Ignition}

Prior to the helium flash, the degenerate He core radiates energy largely by neutrino pair emission. The process is the decay of a plasmon - which one can think of as a photon "dressed" by electron-hole excitations - thereby acquiring an effective mass of about $10 \mathrm{keV}$. The photon couples to a neutrino pair through a electron particle-hole pair that then decays into a $Z_{o} \rightarrow \nu \bar{\nu}$. 
If this cooling is somehow enhanced, the degenerate helium core would be kept cooler, and would not ignite at the normal time. Instead it would continue to grow until it overcame the enhanced cooling to reach, once again, the ignition temperature.

One possible mechanism for enhanced cooling is a neutrino magnetic moment. Then the plasmon could directly couple to a neutrino pair. The strength of this coupling would depend on the size of the magnetic moment.

A delay in the time of He ignition has several observable consequences, including changing the ratio of red giant to horizontal branch stars. Thus, using the standard theory of red giant evolution, investigators have attempted to determine what size of magnetic moment would produce unacceptable changes in the astronomy. The result is a limit 32 on the neutrino magnetic moment of

$$
\mu_{i j} \lesssim 3 \cdot 10^{-12} \text { electron Bohr magnetons }
$$

as was mentioned earlier. This limit is more than two orders of magnitude more stringent than that from direct laboratory tests.

This example is just one of a number of such constraints that can be extracted from similar stellar cooling arguments. The arguments above, for example, can be repeated for neutrino electric dipole moments. More interesting, it can be repeated for axion emission from red giants. Axions, the pseudoGolstone bosons associate with the solution of the strong CP problem suggested by Peccei and Quinn, are very light and can be produced radiatively within the red giant by the Compton process, by the Primakoff process off nuclei, or by emission from low-lying nuclear levels, such at from the $14 \mathrm{keV}$ transition in ${ }^{57} \mathrm{Fe}$. The net result is that axions of mass above a few $\mathrm{eV}$ are excluded; if axions have a direct coupling to electrons, so that the Compton process off electrons operates, the constraint is considerably tighter.

A similar argument can be formulated for supernova cooling. During SN1987A the neutrino burst detected by IMB and by Kamiokande was consistent with cooling on a timescale of about 4 seconds. Thus any process cooling the star more efficiently than neutrino emission would have shortened this time, while also reducing the flux in neutrinos. Large Dirac neutrino masses allow trapped neutrinos to scatter into sterile right-handed states. Right-handed neutrinos, lacking standard model interactions, would then escape the star (provided they do not scatter back into interacting left-handed states). Unfortunately the upper bounds imposed on the neutrino mass are quite model dependent, ranging over (1-25) keV.

The supernova cooling argument can also be repeated for axions. The window of sensitive runs from $1 \mathrm{eV}$ (above this mass they are more strongly coupled than neutrinos, and thus cannot compete with neutrino cooling) to 
about $0.01 \mathrm{eV}$ (below this mass they are too weakly coupled to be produced on the timescale of supernova cooling). It is interesting that the supernova and red giant cooling limit on axions nearly meet: a small window may still exist around a few $\mathrm{eV}$ if the axion has no coupling to electrons.

\section{Supernovae, Supernova Neutrinos, and Nucleosynthesis}

Consider a massive star, in excess of 10 solar masses, burning the hydrogen in its core under the conditions of hydrostatic equilibrium. When the hydrogen is exhausted, the core contracts until the density and temperature are reached where $3 \alpha \rightarrow{ }^{12} \mathrm{C}$ can take place. The He is then burned to exhaustion. This pattern (fuel exhaustion, contraction, and ignition of the ashes of the previous burning cycle) repeats several times, leading finally to the explosive burning of ${ }^{28} \mathrm{Si}$ to Fe. For a heavy star, the evolution is rapid: the star has to work harder to maintain itself against its own gravity, and therefore consumes its fuel faster. A 25 solar mass star would go through all of these cycles in about $7 \mathrm{My}$, with the final explosive Si burning stage taking a few days. The result is an "onion skin" structure of the precollapse star in which the star's history can be read by looking at the surface inward: there are concentric shells of $\mathrm{H}$, ${ }^{4} \mathrm{He},{ }^{12} \mathrm{C},{ }^{16} \mathrm{O}$ and ${ }^{20} \mathrm{Ne},{ }^{28} \mathrm{Si}$, and ${ }^{56} \mathrm{Fe}$ at the center.

\subsection{The Explosion Mechanism 35}

The source of energy for this evolution is nuclear binding energy. A plot of the nuclear binding energy $\delta$ as a function of nuclear mass shows that the minimum is achieved at Fe. In a scale where the ${ }^{12} \mathrm{C}$ mass is picked as zero:

$$
\begin{array}{cc}
{ }^{12} \mathrm{C} & \delta / \text { nucleon }=0.000 \mathrm{MeV} \\
{ }^{16} \mathrm{O} & \delta / \text { nucleon }=-0.296 \mathrm{MeV} \\
{ }^{28} \mathrm{Si} & \delta / \text { nucleon }=-0.768 \mathrm{MeV} \\
{ }^{40} \mathrm{Ca} & \delta / \text { nucleon }=-0.871 \mathrm{MeV} \\
{ }^{56} \mathrm{Fe} & \delta / \text { nucleon }=-1.082 \mathrm{MeV} \\
{ }^{72} \mathrm{Ge} & \delta / \text { nucleon }=-1.008 \mathrm{MeV} \\
{ }^{98} \mathrm{Mo} & \delta / \text { nucleon }=-0.899 \mathrm{Mev}
\end{array}
$$

Thus once the Si burns to produce Fe, there is no further source of nuclear energy adequate to support the star. So as the last remnants of nuclear burning take place, the core is largely supported by degeneracy pressure, with the energy generation rate in the core being less than the stellar luminosity. The core density is about $2 \times 10^{9} \mathrm{~g} / \mathrm{cc}$ and the temperature is $\mathrm{kT} \sim 0.5 \mathrm{MeV}$.

Thus the collapse that begins with the end of Si burning is not halted by a new burning stage, but continues. As gravity does work on the matter, the collapse leads to a rapid heating and compression of the matter. As the 
nucleons in $\mathrm{Fe}$ are bound by about $8 \mathrm{MeV}$, sufficient heating can release $\alpha$ s and a few nucleons. At the same time, the electron chemical potential is increasing. This makes electron capture on nuclei and any free protons favorable,

$$
e^{-}+p \rightarrow \nu_{e}+n
$$

Note that the chemical equilibrium condition is

$$
\mu_{e}+\mu_{p}=\mu_{n}+\left\langle E_{\nu}\right\rangle .
$$

Thus the fact that neutrinos are not trapped plus the rise in the electron Fermi surface as the density increases, lead to increased neutronization of the matter. The escaping neutrinos carry off energy and lepton number. Both the electron capture and the nuclear excitation and disassociation take energy out of the electron gas, which is the star's only source of support. This means that the collapse is very rapid. Numerical simulations find that the iron core of the star $(\sim 1.2-1.5$ solar mases) collapses at about 0.6 of the free fall velocity.

In the early stages of the infall the $\nu_{e}$ s readily escape. But neutrinos are trapped when a density of $\sim 10^{12} \mathrm{~g} / \mathrm{cm}^{3}$ is reached. At this point the neutrinos begin to scatter off the matter through both charged current and coherent neutral current processes. The neutral current neutrino scattering off nuclei is particularly important, as the scattering cross section is off the total nuclear weak charge, which is approximately the neutron number. This process transfers very little energy because the mass energy of the nucleus is so much greater than the typical energy of the neutrinos. But momentum is exchanged. Thus the neutrino "random walks" out of the star. When the neutrino mean free path becomes sufficiently short, the "trapping time" of the neutrino begins to exceed the time scale for the collapse to be completed. This occurs at a density of about $10^{12} \mathrm{~g} / \mathrm{cm}^{3}$, or somewhat less than $1 \%$ of nuclear density. After this point, the energy released by further gravitational collapse and the star's remaining lepton number are trapped within the star.

If we take a neutron star of 1.4 solar masses and a radius of $10 \mathrm{~km}$, an estimate of its binding energy is

$$
\frac{G M^{2}}{2 R} \sim 2.5 \times 10^{53} \mathrm{ergs} .
$$

Thus this is roughly the trapped energy that will later be radiated in neutrinos.

The trapped lepton fraction $Y_{L}$ is a crucial parameter in the explosion physics: a higher trapped $Y_{L}$ leads to a larger homologous core, a stronger shock wave, and easier passage of the shock wave through the outer core, as will be discussed below. Most of the lepton number loss of an infalling mass 
element occurs as it passes through a narrow range of densities just before trapping. The reasons for this are relatively simple: on dimensional grounds weak rates in a plasma go as $T^{5}$, where $\mathrm{T}$ is the temperature. Thus the electron capture rapidly turns on as matter falls toward the trapping radius, and lepton number loss is maximal just prior to trapping. Inelastic neutrino reactions have an important effect on these losses, as the coherent trapping cross section goes as $E_{\nu}^{2}$ and is thus least effective for the lowest energy neutrinos. As these neutrinos escape, inelastic reactions repopulate the low energy states, allowing the neutrino emission to continue.

The velocity of sound in matter rises with increasing density. The inner homologous core, with a mass $M_{H C} \sim 0.6-0.9$ solar masses, is that part of the iron core where the sound velocity exceeds the infall velocity. This allows any pressure variations that may develop in the homologous core during infall to even out before the collapse is completed. As a result, the homologous core collapses as a unit, retaining its density profile. That is, if nothing were to happen to prevent it, the homologous core would collapse to a point.

The collapse of the homologous core continues until nuclear densities are reached. As nuclear matter is rather incompressible $\left(\sim 200 \mathrm{MeV} / \mathrm{f}^{3}\right)$, the nuclear equation of state is effective in halting the collapse: maximum densities of 3-4 times nuclear are reached, e.g., perhaps $6 \cdot 10^{14} \mathrm{~g} / \mathrm{cm}^{3}$. The innermost shell of matter reaches this supernuclear density first, rebounds, sending a pressure wave out through the homologous core. This wave travels faster than the infalling matter, as the homologous core is characterized by a sound speed in excess of the infall speed. Subsequent shells follow. The resulting series of pressure waves collect near the sonic point (the edge of the homologous core). As this point reaches nuclear density and comes to rest, a shock wave breaks out and begins its traversal of the outer core.

Initially the shock wave may carry an order of magnitude more energy than is needed to eject the mantle of the star (less than $10^{51} \mathrm{ergs}$ ). But as the shock wave travels through the outer iron core, it heats and melts the iron that crosses the shock front, at a loss of $\sim 8 \mathrm{MeV} /$ nucleon. The enhanced electron capture that occurs off the free protons left in the wake of the shock, coupled with the sudden reduction of the neutrino opacity of the matter (recall $\sigma_{\text {coherent }} \sim N^{2}$ ), greatly accelerates neutrino emission. This is another energy loss. [Many numerical models predict a strong "breakout" burst of $\nu_{e} \mathrm{~s}$ in the few milliseconds required for the shock wave to travel from the edge of the homologous core to the neutrinosphere at $\rho \sim 10^{12} \mathrm{~g} / \mathrm{cm}^{3}$ and $r \sim 50 \mathrm{~km}$. The neutrinosphere is the term from the neutrino trapping radius, or surface of last scattering.] The summed losses from shock wave heating and neutrino emission are comparable to the initial energy carried by the shock wave. Thus 
most numerical models fail to produce a successful "prompt" hydrodynamic explosion.

Most of the attention in the past decade focused on two explosion scenarios. In the prompt mechanism described above, the shock wave is sufficiently strong to survive the passage of the outer iron core with enough energy to blow off the mantle of the star. The most favorable results were achieved with smaller stars (less than 15 solar masses) where there is less overlying iron, and with soft equations of state, which produce a more compact neutron star and thus lead to more energy release. In part because of the lepton number loss problems discussed earlier, now it is widely believed that this mechanism fails for all but unrealistically soft nuclear equations of state.

The delayed mechanism begins with a failed hydrodynamic explosion; after about 0.01 seconds the shock wave stalls at a radius of $200-300 \mathrm{~km}$. It exists in a sort of equilibrium, gaining energy from matter falling across the shock front, but loosing energy to the heating of that material. However, after perhaps 0.5 seconds, the shock wave is revived due to neutrino heating of the nucleon "soup" left in the wake of the shock. This heating comes primarily from charged current reactions off the nucleons in that nucleon gas; quasielastic scattering also contributes. This high entropy radiation-dominated gas may reach two $\mathrm{MeV}$ in temperature. The pressure exerted by this gas helps to push the shock outward. It is important to note that there are limits to how effective this neutrino energy transfer can be: if matter is too far from the core, the coupling to neutrinos is too weak to deposite significant energy. If too close, the matter may be at a temperature (or soon reach a temperature) where neutrino emission cools the matter as fast or faster than neutrino absorption heats it. The term "gain radius" is used to describe the region where useful heating is done.

This subject is still controversial and unclear. The problem is numerically challenging, forcing modelers to handle the difficult hydrodynamics of a shock wave; the complications of the nuclear equation of state at densities not yet accessible to experiment; modeling in two or three dimensions; handling the slow diffusion of neutrinos; etc. Not all of these aspects can be handled reasonably at the same time, even with existing supercomputers. Thus there is considerable disagreement about whether we have any supernova model that succeeds in ejecting the mantle.

However the explosion proceeds, there is agreement that $99 \%$ of the $3 \cdot 10^{53}$ ergs released in the collapse is radiated in neutrinos of all flavors. The time scale over which the trapped neutrinos leak out of the protoneutron star is about three seconds. Through most of their migration out of the protoneutron 
star, the neutrinos are in flavor equilibrium

$$
\text { e.g., } \quad \nu_{\mathrm{e}}+\bar{\nu}_{\mathrm{e}} \leftrightarrow \nu_{\mu}+\bar{\nu}_{\mu} .
$$

As a result, there is an approximate equipartition of energy among the neutrino flavors. After weak decoupling, the $\nu_{e} \mathrm{~s}$ and $\overline{\nu_{e}}$ s remain in equilibrium with the matter for a longer period than their heavy-flavor counterparts, due to the larger cross sections for scattering off electrons and because of the chargecurrent reactions

$$
\begin{aligned}
\nu_{e}+n & \leftrightarrow p+e^{-} \\
\overline{\nu_{e}}+p & \leftrightarrow n+e^{+} .
\end{aligned}
$$

Thus the heavy flavor neutrinos decouple from deeper within the star, where temperatures are higher. Typical calculations yield

$$
T_{\nu_{\mu}} \sim T_{\nu_{\tau}} \sim 8 \mathrm{MeV} \quad \mathrm{T}_{\nu_{\mathrm{e}}} \sim 3.5 \mathrm{MeV} \quad \mathrm{T}_{\overline{\nu_{\mathrm{e}}}} \sim 4.5 \mathrm{MeV} .
$$

The difference between the $\nu_{e}$ and $\overline{\nu_{e}}$ temperatures is a result of the neutron richness of the matter, which enhances the rate for charge-current reactions of the $\nu_{e} \mathrm{~s}$, thereby keeping them coupled to the matter somewhat longer.

This temperature hierarchy is crucially important to nucleosynthesis and also to possible neutrino oscillation scenarios. The three-flavor MSW levelcrossing diagram is shown in Fig. 10. One very popular scenario attributes the solar neutrino problem to $\nu_{\mu} \leftrightarrow \nu_{e}$ transmutation; this means that a second crossing with a $\nu_{\tau}$ could occur at higher density. It turns out plausible seasaw mass patterns suggest a $\nu_{\tau}$ mass on the order of a few $\mathrm{eV}$, which would be interesting cosmologically. The second crossing would then occur outside the neutrino sphere, that is, after the neutrinos have decoupled and have fixed spectra with the temperatures given above. Thus a $\nu_{e} \leftrightarrow \nu_{\tau}$ oscillation would produce a distinctive $T \sim 8 \mathrm{MeV}$ spectrum of $\nu_{e}$ s. This has dramatic consequences for terrestrial detection and for nucleosynthesis in the supernova.

\subsection{The Neutrino Process 36}

Core-collapse supernovae are one of the major engines driving galactic chemical evolution, producing and ejecting the metals that enrich our galaxy. The discussion of the previous section described the hydrostatic evolution of a presupernova star in which large quantities of the most abundant metals $(\mathrm{C}, \mathrm{O}, \mathrm{Ne}$, ...) are synthesized and later ejected during the explosion. During the passage of the shock wave through the star's mantle, temperature of $\sim(1-3) \cdot 10^{9} \mathrm{~K}$ and are reached in the silicon, oxygen, and neon shells. This shock wave heating 


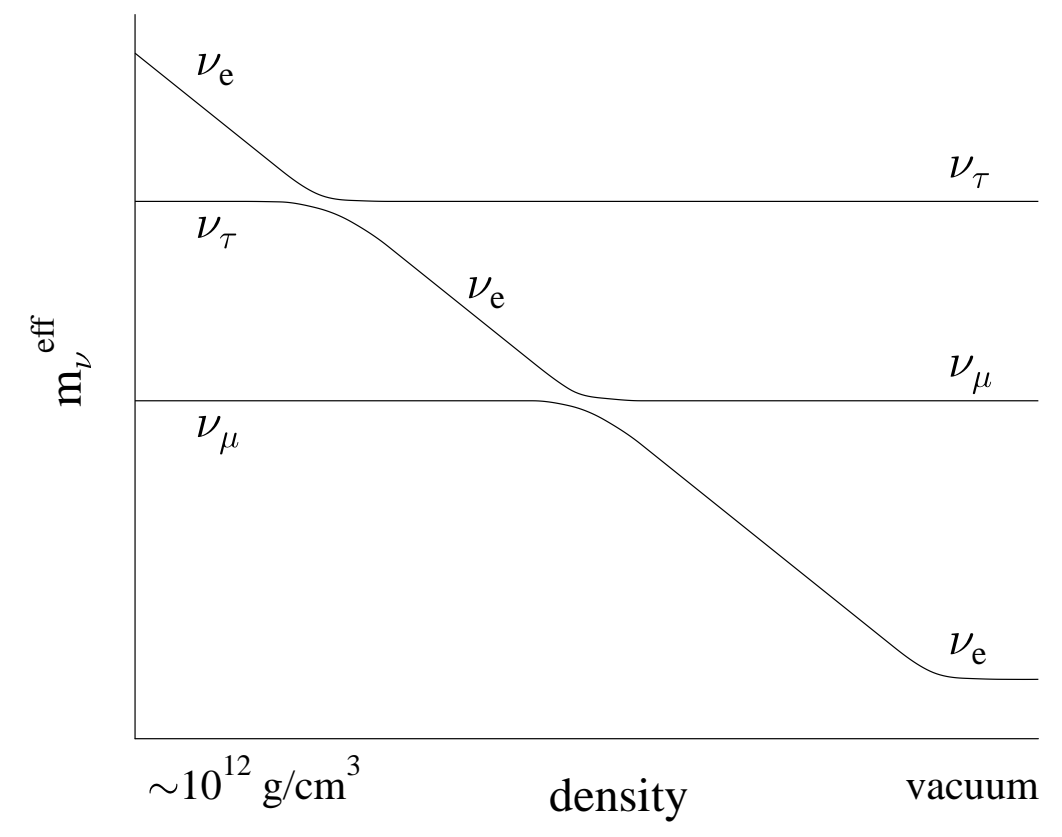

Figure 10: Three-flavor neutrino level-crossing diagram. One popular scenario associates the solar neutrino problem with $\nu_{e} \leftrightarrow \nu_{\mu}$ oscillations and predicts a cosmologically interested massive $\nu_{\tau}$ with $\nu_{e} \leftrightarrow \nu_{\tau}$ oscillations near the supernova neutrinosphere.

induces $(\gamma, \alpha) \leftrightarrow(\alpha, \gamma)$ and related reactions that generate a mass flow toward highly bound nuclei, resulting in the synthesis of iron peak elements as well as less abundant odd-A species. Rapid neutron-induced reactions are thought to take place in the high-entropy atmosphere just above the mass cut, producing about half of the heavy elements above $\mathrm{A} \sim 80$. This is the subject of the Sec. 4.3. Finally, the $\nu$-process described below is responsible for the synthesis of rare species such as ${ }^{11} \mathrm{~B}$ and ${ }^{19} \mathrm{~F}$. This process involves the response of nuclei at momentum transfers where the allowed approximation is no longer valid. Thus we will use the $\nu$-process in this section to illustrate some of the relevant nuclear physics.

One of the problems - still controversial - that may be connected with the neutrino process is the origin of the light elements $\mathrm{Be}, \mathrm{B}$ and $\mathrm{Li}$, elements which are not produced in sufficient amounts in the big bang or in any of the stellar mechanisms we have discussed. The traditional explanation has been cosmic 
ray spallation interactions with $\mathrm{C}, \mathrm{O}$, and $\mathrm{N}$ in the interstellar medium. In this picture, cosmic ray protons collide with $\mathrm{C}$ at relatively high energy, knocking the nucleus apart. So in the debris one can find nuclei like ${ }^{10} \mathrm{~B},{ }^{11} \mathrm{~B}$, and ${ }^{7} \mathrm{Li}$.

But there are some problems with this picture. First of all, this is an example of a secondary mechanism: the interstellar medium must be enriched in the $\mathrm{C}, \mathrm{O}$, and $\mathrm{N}$ to provide the targets for these reactions. Thus cosmic ray spallation must become more effective as the galaxy ages. The abundance of boron, for example, would tend to grow quadratically with metalicity, since the rate of production goes linearly with metalicity. But obseryations, especially recent measurements with the HST, find a linear growth 37 in the boron abundance.

A second problem is that the spectrum of cosmic ray protons peaks near $1 \mathrm{GeV}$, leading to roughly comparable production of the two isotopes ${ }^{10} \mathrm{~B}$ and ${ }^{11} \mathrm{~B}$. That is, while it takes more energy to knock two nucleons out of carbon than one, this difference is not significant compared to typical cosmic ray energies. More careful studies lead to the expectation that the abundance ratio of ${ }^{11} \mathrm{~B}$ to ${ }^{10} \mathrm{~B}$ might be $\sim 2$. In nature, it is greater than 4 .

Fans of cosmic ray spallation have offered solutions to these problems, e.g., similar reactions occurring in the atmospheres of nebulae involving lower energy cosmic rays. As this suggestion was originally stimulated by the observation of nuclear $\gamma$ rays from Orion, now retracted, some of the motivation for this scenario has evaporated. Here I focus on an alternative explanation, synthesis via neutrino spallation.

Previously we described the allowed Gamow-Teller (spin-flip) and Fermi weak interaction operators. These are the appropriate operators when one probes the nucleus at a wavelength - that is, at a size scale - where the nucleus responds like an elementary particle. We can then characterize its response by its macroscopic quantum numbers, the spin and charge. On the other hand, the nucleus is a composite object and, therefore, if it is probed at shorter length scales, all kinds of interesting radial excitations will result, analogous to the vibrations of a drumhead. For a reaction like neutrino scattering off a nucleus, the full operator involves the additional factor

$$
e^{i \vec{k} \cdot \vec{r}} \sim 1+i \vec{k} \cdot \vec{r}
$$

where the expression on the right is valid if the magnitude of $\vec{k}$ is not too large. Thus the full charge operator includes a "first forbidden" term

$$
\sum_{i=1}^{A} \vec{r}_{i} \tau_{3}(i)
$$


and similarly for the spin operator

$$
\sum_{i=1}^{A}\left[\vec{r}_{i} \otimes \vec{\sigma}(i)\right]_{J=0,1,2} \tau_{3}(i) .
$$

These operators generate collective radial excitations, leading to the so-called "giant resonance" excitations in nuclei. The giant resonances are typically at an excitation energy of $20-25 \mathrm{MeV}$ in light nuclei. One important property is that these operators satisfy a sum rule (Thomas-Reiche-Kuhn) of the form

$$
\sum_{f}\left|\left\langle f\left|\sum_{i=1}^{A} r(i) \tau_{3}(i)\right| i\right\rangle\right|^{2} \sim \frac{N Z}{A} \sim \frac{A}{4}
$$

where the sum extends over a complete set of final nuclear states. These first-forbidden operators tend to dominate the cross sections for scattering the high energy supernova neutrinos $\left(\nu_{\mu} \mathrm{s}\right.$ and $\left.\nu_{\tau} \mathrm{s}\right)$, with $E_{\nu} \sim 25 \mathrm{MeV}$, off light nuclei. From the sum rule above, it follows that nuclear cross sections per target nucleon are roughly constant.

The E1 giant dipole mode described above is depicted qualitatively in Fig. 11a. This description, which corresponds to an early model of the giant resonance response by Goldhaber and Teller, involves the harmonic oscillation of the proton and neutron fluids against one another. The restoring force for small displacements would be linear in the displacement and dependent on the nuclear symmetry energy. There is a natural extension of this model to weak interactions, where axial excitations occur. For example, one can envision a mode similar to that of Fig. 11a where the spin-up neutrons and spin-down protons oscillate against spin-down neutrons and spin-up protons, the spin-isospin mode of Fig. 11b. This mode is one that arises in a simple SU(4) extension of the Goldhaber-Teller model, derived by assuming that the nuclear force is spin and isospin independent, at the same excitation energy as the E1 mode. In full, the Goldhaber-Teller model predicts a degenerate 15-dimensional supermultiplet of giant resonances, each obeying sum rules analogous to the TRK sum rule. While more sophisticated descriptions of the giant resonance region are available, of course, this crude picture is qualitatively accurate.

This nuclear physics is important to the $\nu$-process. The simplest example of $\nu$-process nucleosynthesis involves the Ne shell in a supernova. Because of the first-forbidden contributions, the cross section for inelastic neutrino scattering to the giant resonances in Ne is $\sim 3 \cdot 10^{-41} \mathrm{~cm}^{2} /$ flavor for the more energetic heavy-flavor neutrinos. This reaction

$$
\nu+A \rightarrow \nu^{\prime}+A^{*}
$$


a)

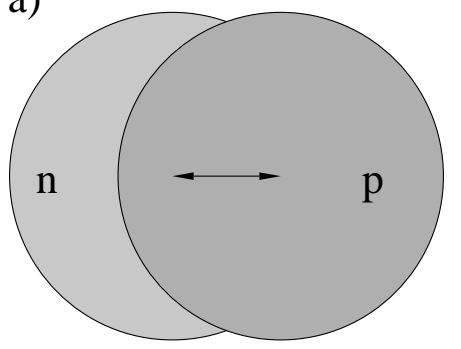

b)

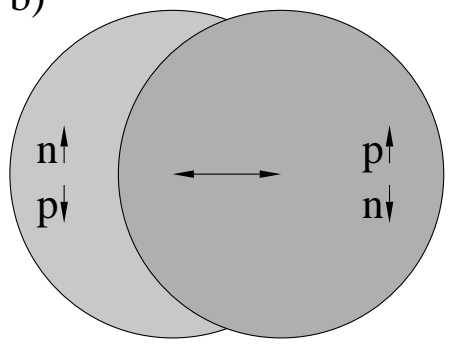

Figure 11: Schematic illustration of a) the E1 giant dipole mode familiar from electromagnetic interactions and b) a spin-isospin giant dipole mode associated with the first-forbidden weak axial response.

transfers an energy typical of giant resonances, $\sim 20 \mathrm{MeV}$. A supernova releases about $3 \times 10^{53}$ ergs in neutrinos, which converts to about $4 \times 10^{57}$ heavy flavor neutrinos. The Ne shell in a $20 \mathrm{M}_{\odot}$ star has at a radius $\sim 20,000 \mathrm{~km}$. Thus the neutrino fluence through the Ne shell is

$$
\phi \sim \frac{4 \cdot 10^{57}}{4 \pi(20,000 \mathrm{~km})^{2}} \sim 10^{38} / \mathrm{cm}^{2} .
$$

Thus folding the fluence and cross section, one concludes that approximately $1 / 300$ th of the Ne nuclei interact.

This is quite interesting since the astrophysical origin of ${ }^{19} \mathrm{~F}$ had not been understood. The only stable isotope of fluorine, ${ }^{19} \mathrm{~F}$ has an abundance

$$
\frac{{ }^{19} \mathrm{~F}}{{ }^{20} \mathrm{Ne}} \sim \frac{1}{3100} .
$$

This leads to the conclusion that the fluorine found in toothpaste was created by neutral current neutrino reactions deep inside some ancient supernova.

The calculation of the final ${ }^{19} \mathrm{~F} /{ }^{20} \mathrm{Ne}$ ratio is more complicated than the simple 1/300 ratio given above:

- When $\mathrm{Ne}$ is excited by $\sim 20 \mathrm{MeV}$ through inelastic neutrino scattering, it breaks up in two ways

$$
\begin{aligned}
& { }^{20} \mathrm{Ne}\left(\nu, \nu^{\prime}\right)^{20} \mathrm{Ne}^{*} \rightarrow{ }^{19} \mathrm{Ne}+\mathrm{n} \rightarrow{ }^{19} \mathrm{~F}+\mathrm{e}^{+}+\nu_{\mathrm{e}}+\mathrm{n} \\
& { }^{20} \mathrm{Ne}\left(\nu, \nu^{\prime}\right)^{20} \mathrm{Ne}^{*} \rightarrow{ }^{19} \mathrm{~F}+\mathrm{p}
\end{aligned}
$$


with the first reaction occurring half as frequently as the second. As both channels lead to ${ }^{19} \mathrm{~F}$, we have correctly estimated the instantaneous abundance ratio in the Ne shell of

$$
\frac{{ }^{19} \mathrm{~F}}{{ }^{20} \mathrm{Ne}} \sim \frac{1}{300} .
$$

- We must also address the issue of whether the produced ${ }^{19} \mathrm{~F}$ survives. In the first $10^{-8}$ sec the coproduced neutrons in the first reaction react via

$$
{ }^{15} \mathrm{O}(\mathrm{n}, \mathrm{p}){ }^{15} \mathrm{~N} \quad{ }^{19} \mathrm{Ne}(\mathrm{n}, \alpha){ }^{16} \mathrm{O} \quad{ }^{20} \mathrm{Ne}(\mathrm{n}, \gamma){ }^{21} \mathrm{Ne}{ }^{19} \mathrm{Ne}(\mathrm{n}, \mathrm{p}){ }^{19} \mathrm{~F}
$$

with the result that about $70 \%$ of the ${ }^{19} \mathrm{~F}$ produced via spallation of neutrons is then immediate destroyed, primarily by the $(n, \alpha)$ reaction above. In the next $10^{-6}$ sec the coproduced protons are also processed

$$
{ }^{15} \mathrm{~N}(\mathrm{p}, \alpha){ }^{12} \mathrm{C}{ }^{19} \mathrm{~F}(\mathrm{p}, \alpha){ }^{16} \mathrm{O}{ }^{23} \mathrm{Na}(\mathrm{p}, \alpha){ }^{20} \mathrm{Ne}
$$

with the latter two reactions competing as the primary proton poisons. This makes an important prediction: stars with high $\mathrm{Na}$ abundances should make more $\mathrm{F}$, as the ${ }^{23} \mathrm{Na}$ acts as a proton poison to preserve the produced $\mathrm{F}$.

- Finally, there is one other destruction mechanism, the heating associated with the passage of the shock wave. It turns out the the F produced prior to shock wave passage can survive if it is in the outside half of the Ne shell. The reaction

$$
{ }^{19} \mathrm{~F}(\gamma, \alpha){ }^{15} \mathrm{~N}
$$

destroys $\mathrm{F}$ for peak explosion temperatures exceeding $1.7 \cdot 10^{9} \mathrm{~K}$. Such a temperature is produced at the inner edge of the Ne shell by the shock wave heating, but not at the outer edge.

If all of this physics in handled is a careful network code that includes the shock wave heating and $\mathrm{F}$ production both before and after shock wave passage, the following are the results:

$\begin{array}{cc}\frac{\left[{ }^{19} \mathrm{~F} /{ }^{20} \mathrm{Ne}\right] /\left[{ }^{19} \mathrm{~F} /{ }^{20} \mathrm{Ne}\right]_{\odot}}{0.14} & \frac{T_{\text {heavy } \nu}(\mathrm{MeV})}{4} \\ 0.6 & 6 \\ 1.2 & 8 \\ 1.1 & 10 \\ 1.1 & 12\end{array}$

where the abundance ratio in the first column has been normalized to the solar value. One sees that the attribution of $\mathrm{F}$ to the neutrino process argues that the heavy flavor $\nu$ temperature must be greater than $6 \mathrm{MeV}$, a result theory 
favors. One also sees that $\mathrm{F}$ cannot be overproduced by this mechanism: although the instantaneous production of $\mathrm{F}$ continues to grow rapidly with the neutrino temperature, too much $\mathrm{F}$ results in its destruction through the $(p, \alpha)$ reaction, given a solar abundance of the competing proton poison ${ }^{23} \mathrm{Na}$. Indeed, this illustrates an odd quirk: although in most cases the neutrino process is a primary mechanism, one needs ${ }^{23} \mathrm{Na}$ present to produce significant $\mathrm{F}$. Thus in this case the neutrino process is a secondary mechanism.

While there are other significant neutrino process products $\left({ }^{7} \mathrm{Li},{ }^{138} \mathrm{La}\right.$, ${ }^{180} \mathrm{Ta},{ }^{15} \mathrm{~N} \ldots$...), the most important product is ${ }^{11} \mathrm{~B}$, produced by spallation off carbon. A calculation by Timmes et al. [18] found that the combination of the neutrino process, cosmic ray spallation and big-bang nucleosythesis together can explain the evolution of the light elements. The neutrino process, which produces a great deal of ${ }^{11} \mathrm{~B}$ but relatively little ${ }^{10} \mathrm{~B}$, combines with the cosmic ray spallation mechanism to yield the observed isotope ratio. Again, one prediction of this picture is that early stars should be ${ }^{11} \mathrm{~B}$ rich, as the neutrino process is primary and operates early in our galaxy's history; the cosmic ray production of ${ }^{10} \mathrm{~B}$ is more recent. There is hope that HST studies will soon be able to descriminate between ${ }^{10} \mathrm{~B}$ and ${ }^{11} \mathrm{~B}$ : as yet this has not been done.

\subsection{The r-process}

Beyond the iron peak nuclear Coulomb barriers become so high that charged particle reactions become ineffective, leaving neutron capture as the mechanism responsible for producing the heaviest nuclei. If the neutron abundance is modest, this capture occurs in such a way that each newly synthesized nucleus has the opportunity to $\beta$ decay, if it is energetically favorable to do so. Thus weak equilibrium is maintained within the nucleus, so that synthesis is along the path of stable nuclei. This is called the s- or slow-process. However a plot of the s-process in the $(\mathrm{N}, \mathrm{Z})$ plane reveals that this path misses many stable, neutron-rich nuclei that are known to exist in nature. This suggests that another mechanism is at work, too. Furthermore, the abundance peaks found in nature near masses $\mathrm{A} \sim 130$ and $\mathrm{A} \sim 190$, which mark the closed neutron shells where neutron capture rates and $\beta$ decay rates are slower, each split into two subpeaks. One set of subpeaks corresponds to the closed-neutron-shell numbers $\mathrm{N} \sim 82$ and $\mathrm{N} \sim 126$, and is clearly associated with the s-process. The other set is shifted to smaller $\mathrm{N}, \sim 76$ and $\sim 116$, respectively, and is suggestive of a much more explosive neutron capture environment where neutron capture can be rapid.

This second process is the r- or rapid-process, characterized by:

- The neutron capture is fast compared to $\beta$ decay rates. 
- The equilibrium maintained within a nucleus is established by $(n, \gamma) \leftrightarrow(\gamma, n)$ : neutron capture fills up the available bound levels in the nucleus until this equilibrium sets in. The new Fermi level depends on the temperature and the relative $n / \gamma$ abundance.

- The nucleosynthesis rate is thus controlled by the $\beta$ decay rate: each $\beta^{-}$ capture coverting $\mathrm{n} \rightarrow \mathrm{p}$ opens up a hole in the neutron Fermi sea, allowing another neutron to be captured.

- The nucleosynthesis path is along exotic, neutron-rich nuclei that would be highly unstable under normal laboratory conditions.

- As the nucleosynthesis rate is controlled by the $\beta$ decay, mass will build up at nuclei where the $\beta$ decay rates are slow. It follows, if the neutron flux is reasonable steady over time so that equilibrated mass flow is reached, that the resulting abundances should be inversely proportional to these $\beta$ decay rates.

Let's first explore the $(n, \gamma) \leftrightarrow(\gamma, n)$ equilibrium condition, which requires that the rate for $(n, \gamma)$ balances that for $(\gamma, n)$ for an average nucleus. So consider the formation cross section

$$
A+n \rightarrow(A+1)+\gamma .
$$

This is an exothermic reaction, as the neutron drops into the nuclear well. Our averaged cross section, assuming a resonant reaction (the level density is high in heavy nuclei) is

$$
\langle\sigma v\rangle_{(n, \gamma)}=\left(\frac{2 \pi}{\mu k T}\right)^{3 / 2} \frac{\Gamma_{n} \Gamma_{\gamma}}{\Gamma} e^{-E / K T}
$$

where $\mathrm{E} \sim 0$ is the resonance energy, and the $\Gamma \mathrm{s}$ are the indicated partial and total widths. Thus the rate per unit volume is

$$
r_{(n, \gamma)} \sim N_{n} N_{A}\left(\frac{2 \pi}{\mu k T}\right)^{3 / 2} \frac{\Gamma_{n} \Gamma_{\gamma}}{\Gamma}
$$

where $N_{n}$ and $N_{A}$ are the neutron and nuclear number densities and $\mu$ the reduced mass. This has to be compared to the $(\gamma, n)$ rate.

The $(\gamma, n)$ reaction requires the photon number density in the gas. This is given by the Bose-Einstein distribution

$$
N(\epsilon)=\frac{8 \pi}{c^{3} h^{3}} \frac{\epsilon^{2} d \epsilon}{e^{\epsilon / k T}-1} .
$$

The high-energy tail of the normalized distribution can thus be written

$$
\sim \frac{1}{N_{\gamma} \pi^{2}} \epsilon^{2} e^{-\epsilon / k T} d \epsilon
$$


where in the last expression we have set $\hbar=c=1$.

Now we need the resonant cross section in the $(\gamma, n)$ direction. For photons the wave number is proportional to the energy, so

$$
\sigma_{(\gamma, n)}=\frac{\pi}{\epsilon^{2}} \frac{\Gamma_{\gamma} \Gamma_{n}}{\left(\epsilon-E_{r}\right)^{2}+(\Gamma / 2)^{2}} .
$$

As the velocity is $\mathrm{c}=1$,

$$
\langle\sigma v\rangle=\frac{1}{\pi^{2} N_{\gamma}} \int_{0}^{\infty} \epsilon^{2} e^{-\epsilon / k T} d \epsilon \frac{\pi}{\epsilon^{2}} \frac{\Gamma_{\gamma} \Gamma_{n}}{\left(\epsilon-E_{r}\right)^{2}+(\Gamma / 2)^{2}} .
$$

We evaluate this in the usual way for a sharp resonance, remembering that the energy integral over just the denominator above (the sharply varying part) is $2 \pi / \Gamma$

$$
\sim \frac{\Gamma_{\gamma} \Gamma_{n}}{N_{\gamma}} e^{-E_{r} / k T} \frac{2}{\Gamma}
$$

So that the rate becomes

$$
r_{(\gamma, n)} \sim 2 N_{A+1} \frac{\Gamma_{\gamma} \Gamma_{n}}{\Gamma} e^{-E_{r} / k T} .
$$

Equating the $(n, \gamma)$ and $(\gamma, n)$ rates and taking $N_{A} \sim N_{A-1}$ then yields

$$
N_{n} \sim \frac{2}{(\hbar c)^{3}}\left(\frac{\mu c^{2} k T}{2 \pi}\right)^{3 / 2} e^{-E_{r} / k T}
$$

where the $\hbar \mathrm{s}$ and $c \mathrm{~s}$ have been properly inserted to give the right dimensions. Now $E_{r}$ is esssentially the binding energy. So plugging in the conditions $N_{n} \sim$ $3 \times 10^{23} / \mathrm{cm}^{3}$ and $T_{9} \sim 1$, we find that the binding energy is $\sim 2.4 \mathrm{MeV}$. Thus neutrons are bound by about 30 times $k T$, a value that is still small compared to a typical binding of $8 \mathrm{MeV}$ for a normal nucleus. (In this calculation I calculated the neutron reduced mass assuming a nuclear target with $\mathrm{A}=150$.)

The above calculation fails to count spin states for the photons and nuclei and is thus not quite correct. But it makes the essential point: the r-process involves very exotic species largely unstudied in any terrestrial laboratory. It is good to bear this in mind, as in the following section we will discuss the responses of such nuclei to neutrinos. Such responses thus depend on the ability of theory to extrapolate responses from known nuclei to those quite unfamiliar.

The path of the r-process is along neutron-rich nuclei, where the neutron Fermi sea is just $\sim(2-3) \mathrm{MeV}$ away from the neutron drip line (where no 
more bound neutron levels exist). After the r-process finishes (the neutron exposure ends) the nuclei decay back to the valley of stability by $\beta$ decay. This can involve some neutron spallation ( $\beta$-delayed neutrons) that shift the mass number A to a lower value. But it certainly involves conversion of neutrons into protons, and that shifts the r-process peaks at $\mathrm{N} \sim 82$ and 126 to a lower $\mathrm{N}$, off course. This effect is clearly seen in the abundance distribution: the r-process peaks are shifted to lower $\mathrm{N}$ relative to the s-process peaks. This is the origin of the second set of "subpeaks" mentioned at the start of the section.

It is believed that the r-process can proceed to very heavy nuclei $(\mathrm{A} \sim$ 270) where it is finally ended by $\beta$-delayed and $n$-induced fission, which feeds matter back into the process at an $\mathrm{A} \sim \mathrm{A}_{\max } / 2$. Thus there may be important cycling effects in the upper half of the r-process distribution.

What is the site(s) of the r-process? This has been debated many years and still remains a controversial subject:

- The r-process requires exceptionally explosive conditions

$$
\rho(\mathrm{n}) \sim 10^{20} \mathrm{~cm}^{-3} \quad \mathrm{~T} \sim 10^{9} \mathrm{~K} \quad \mathrm{t} \sim 1 \mathrm{~s} .
$$

- Both primary and secondary sites proposed. Primary sites are those not requiring preexisting metals. Secondary sites are those where the neutron capture occurs on preexisting s-process seeds.

- Suggested primary sites include the the neutronized atmosphere above the proto-neutron star in a Type II supernova, neutron-rich jets produced in supernova explosions or in neutron star mergers, inhomogeneous big bangs, etc. - Secondary sites, where $\rho(\mathrm{n})$ can be lower for successful synthesis, include the $\mathrm{He}$ and $\mathrm{C}$ zones in Type II supernovae, the red giant He flash, etc.

The balance of evidence favors a primary site, so one requiring no preenrichment of heavy s-process metals. Among the evidence:

1) HST studies of very-metal-poor halo stars: The most important evidence are the recent HST measurements of Cowan, Sneden et al. 38 of very metalpoor stars $([\mathrm{Fe} / \mathrm{H}] \sim-1.7$ to -3.12$)$ where an r-process distribution very much like that of our sun has been seen for $\mathrm{Z} \gtrsim 56$. Furthermore, in these stars the iron content is variable. This suggests that the "time resolution" inherent in these old stars is short compared to galactic mixing times (otherwise Fe would be more constant). The conclusion is that the r-process material in these stars is most likely from one or a few local supernovae. The fact that the distributions match the solar r-process (at least above charge 56) strongly suggests that there is some kind of unique site for the r-process: the solar r-process distribution did not come from averaging over many different kinds of r-process events. Clearly the fact that these old stars are enriched in r-process metals also strongly argues for a primary process: the r-process 
works quite well in an environment where there are few initial s-process metals.

2) There are also fairly good theoretical arguments that a primary r-process occurring in a core-collapse supernova might be viable 39 . First, galactic chemical evolution studies indicate that the growth of r-process elements in the galaxy is consistent with low-mass Type II supernovae in rate and distribution. More convincing is the fact that modelers have shown that the conditions needed for an r-process (very high neutron densities, temperatures of 1-3 billion degrees) might be realized in a supernova. The site is the last material expelled from the supernova, the matter just above the mass cut. When this material is blown off the star initially, it is a very hot neutron-rich, radiation-dominated gas containing neutrons and protons, but an excess of the neutrons. As it expands off the star and cools, the material first goes through a freezeout to $\alpha$ particles, a step that essentially locks up all the protons in this way. Then the $\alpha$ s interact through reactions like

$$
\begin{aligned}
& \alpha+\alpha+\alpha \rightarrow^{12} C \\
& \alpha+\alpha+n \rightarrow^{9} B e
\end{aligned}
$$

to start forming heavier nuclei. Note, unlike the big bang, that the density is high enough to allow such three-body interactions to bridge the mass gaps at $\mathrm{A}=5,8$. The $\alpha$ capture continues up to heavy nuclei, to $\mathrm{A} \sim 80$, in the network calculations. The result is a small number of "seed" nuclei, a large number of $\alpha \mathrm{s}$, and excess neutrons. These neutrons preferentially capture on the heavy seeds to produce an r-process. Of course, what is necessary is to have $\sim 100$ excess neutrons per seed in order to successfully synthesize heavy mass nuclei. Some of the modelers find conditions where this almost happens.

There are some very nice aspects of this site: the amount of matter ejected is about $10^{-5}-10^{-6}$ solar masses, which is just about what is needed over the lifetime of the galaxy to give the integrated r-process metals we see, taking a reasonable supernova rate. But there are also a few problems, especially the fact that with calculated entropies in the nucleon soup above the proto-neutron star, neutron fractions appear to be too low to produce a successful A $\sim 190$ peak. There is some interesting recent work invoking neutrino oscillations to cure this problem: charge current reactions on free protons and neutrons determine the $\mathrm{n} / \mathrm{p}$ ratio in the gas. Then, for example, an oscillation of the type $\nu_{e} \rightarrow \nu_{\text {sterile }}$ can alter this ratio, as it would turn off the $\nu_{e}$ s that destroy neutrons by charged-current reactions. Unfortunately, a full discussion of such possibilities would take us too far afield today.

The nuclear physics of the r-process tells us that the synthesis occurs when the nucleon soup is in the temperature range of $(3-1) \cdot 10^{9} \mathrm{~K}$, which, in the hot 
bubble r-process described above, corresponds to a freezeout radius of (600$100) \mathrm{km}$ and a time $\sim 10$ seconds after core collapse. The neutrino fluence after freezeout (when the temperature has dropped below $10^{9} \mathrm{~K}$ and the r-process stops $)$ is then $\sim(0.045-0.015) \cdot 10^{51} \mathrm{ergs} /(100 \mathrm{~km})$. Thus, after completion of the r-process, the newly synthesized material experiences an intense flux of neutrinos. This brings up the question of whether the neutrino flux could have any effect on the r-process.

\subsection{Neutrinos and the r-process 40}

Rather than describe the exotic effects of neutrino oscillations on the r-process, mentioned briefly above, we will examine standard-model effects that are nevertheless quite interesting. The nuclear physics of this section - neutrino-induced neutron spallation reactions - is also relevant to recently proposed supernova neutrino observatories such as OMNIS and LAND. In contrast to our first discussion of the $\nu$-process in Sec. 4.2, it is apparent that neutrino effects could be much larger in the hot bubble r-process: the synthesis occurs much closer to the star than our Ne radius of 20,000 km: estimates are 600-1000 $\mathrm{km}$. The r-process is completed in about 10 seconds (when the temperature drops to about one billion degrees), but the neutrino flux is still significant as the r-process freezes out. The net result is that the "post-processing" neutrino fluence - the fluence that can alter the nuclear distribution after the r-process is completed - is about 100 times larger than that responsible for fluorine production in the Ne zone. Recalling that $1 / 300$ of the nuclei in the Ne zone interacted with neutrinos, and remembering that the relevant neutrino-nucleus cross sections scale as A, one quickly sees that the probability of a r-process nucleus interacting with the neutrino flux is approximately unity.

Because the hydrodynamic conditions of the r-process are highly uncertain, one way to attack this problem is to work backward in time. We know the final r-process distribution (what nature gives us) and we can calculate neutrino-nucleus interactions relatively well. Thus from the observed r-process distribution (including neutrino postprocessing) we can work backward to find out what the r-process distribution looked like at the point of freezeout. In Figs. 12 and 13, the "real" r-process distribution - that produced at freezeout - is given by the dashed lines, while the solid lines show the effects of the neutrino postprocessing for a particular choice of fluence. The nuclear physics input into these calculations is precisely that previously described: GT and first-forbidden cross sections, with the responses centered at excitation energies consistent with those found in ordinary, stable nuclei, taking into account the observed dependence on $|N-Z|$. 


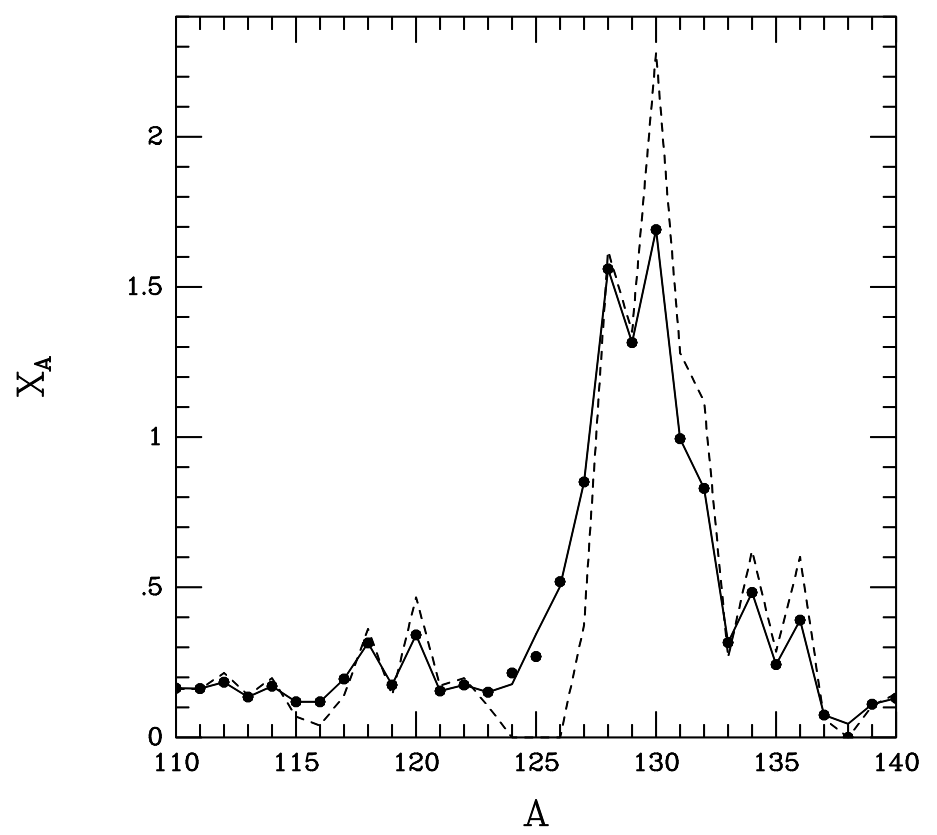

Figure 12: Comparison of the r-process distribution that would result from the freezeout abundances near the $\mathrm{A} \sim 130$ mass peak (dashed line) to that where the effects of neutrino postprocessing have been include (solid line). The fluence has been fixed by assuming that the $\mathrm{A}=124-126$ abundances are entirely due to the $\nu$-process.

One important aspect of the figures is that the mass shift is significant. This has to do with the fact that a $20 \mathrm{MeV}$ excitation of a neutron-rich nucleus allows multiple neutrons $(\sim 5)$ to be emitted. (Remember we found that the binding energy of the last neutron in an r-process neutron-rich nuclei was about 2-3 MeV under typical r-process conditions.) The second thing to notice is that the relative contribution of the neutrino process is particularly important in the "valleys" beneath the mass peaks: the reason is that the parents on the mass peak are abundant, and the valley daughters rare. In fact, it follows from this that the neutrino process effects can be dominant for precisely seven isotopes (Te, Re, etc.) lying in these valleys. Furthermore if an appropriate neutrino fluence is picked, these isotope abundances are produced perfectly (given the abundance errors). The fluences are

$$
\mathrm{N}=82 \text { peak } \quad 0.031 \cdot 10^{51} \mathrm{ergs} /(100 \mathrm{~km})^{2} / \text { flavor }
$$




$$
\mathrm{N}=126 \text { peak } \quad 0.015 \cdot 10^{51} \mathrm{ergs} /(100 \mathrm{~km})^{2} / \text { flavor, }
$$

values in fine agreement with those that would be found in a hot bubble $\mathrm{r}$ process. So this is circumstantial but significant evidence that the material near the mass cut of a Type II supernova is the site of the r-process: there is a neutrino fingerprint.

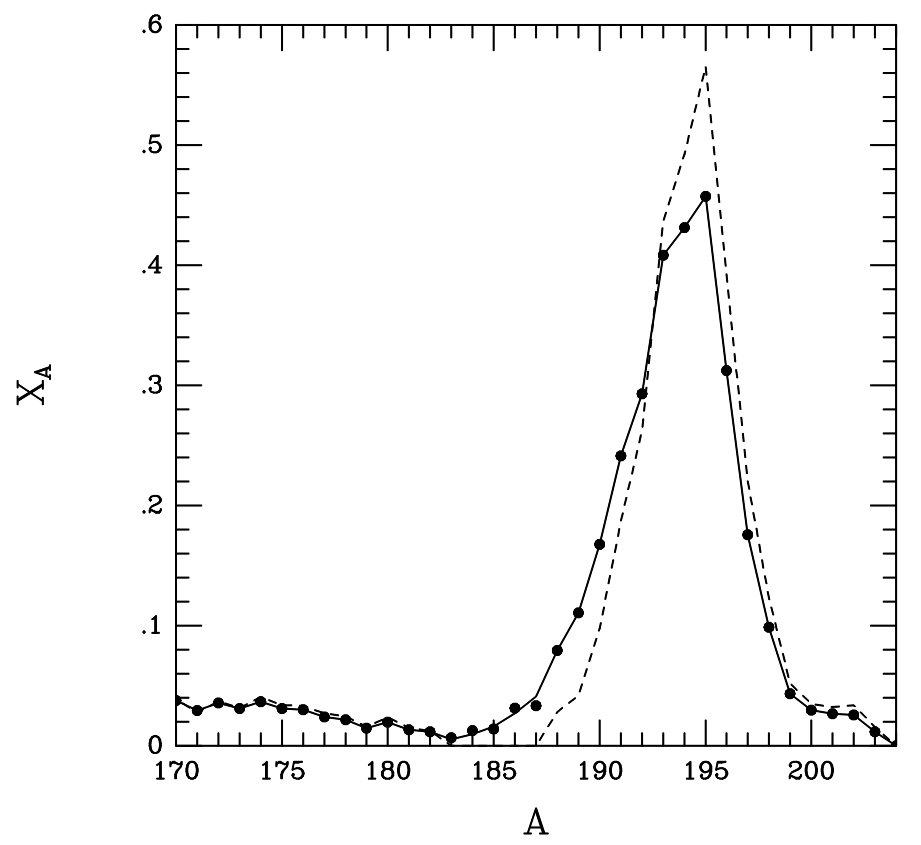

Figure 13: As in Fig. 12, but for the $\mathrm{A} \sim 195$ mass peak. The $\mathrm{A}=183-187$ abundances are entirely attributed to the $\nu$-process.

\subsection{Neutrino Oscillations and the r-process}

For the usual seesaw pattern of neutrino masses and a cosmological interesting $\nu_{\tau}$ (i.e., a heavy neutrino with a mass in the neighborhood of $10 \mathrm{eV}$ ), the full MSW pattern is shown in Fig. 10. If the $\nu_{e}-\nu_{\mu}$ crossing is responsible for the solar neutrino problem, a second crossing, $\nu_{e}-\nu_{\tau}$, is expected at a density large compared to that of the solar core, but small compared to the location of the supernova neutrinosphere $\left(\sim 10^{12} \mathrm{~g} / \mathrm{cm}^{3}\right)$. For a very large range of 
mixing angles, this crossing is adiabatic and thus leads to $\nu_{e} \leftrightarrow \nu_{\tau}$ conversion. These spectra thus change identities, leading to an anomalously hot $\nu_{e}$ flux from a Type II supernova.

As the $\nu$-nucleon cross section is proportional to $\mathrm{E}_{\nu}^{2}$, the reaction $\nu_{e}+\mathrm{n}$ $\rightarrow \mathrm{e}^{-}+\mathrm{p}$ is enhanced, while $\bar{\nu}_{e}+\mathrm{p} \rightarrow \mathrm{e}^{+}+\mathrm{n}$ is unchanged. For a rather extensive range of $\nu_{e} \leftrightarrow \nu_{\tau}$ mixing angles and $\delta m^{2}$, this crossing then destroys the r-process: the hotter $\nu_{e}$ s drive the matter proton rich 41 . Thus, if one accepts this location as the site of the r-process, very strong constraints on cosmologically interesting $\nu_{\tau}$ s are obtained. These limits are truly remarkable for their sensitivity to small mixing angles, extended to $\sin ^{2} 2 \theta \sim 10^{-5}$ for neutrino mass differences above a few $\mathrm{eV}^{2}$.

I thank A. S. Brun and R. E. Shrock for helpful comments, and Paul Langacker for his able organization of the 1998 TASI summer school. This work was supported in part by the US Department of Energy.

\section{References}

1. This section taken from W. C. Haxton, Ann. Rev. Astron. Astrophys. 33, 459 (1995).

2. R. Davis, Jr., D. S. Harmer, and K. C. Hoffman, Phys. Rev. Lett. 20, 1205 (1966).

3. J. N. Abdurashitov et al., Phys. Lett. B 328, 234 (1994).

4. P. Anselmann et al., Phys. Lett. B 285, 376 (1992).

5. Y. Suzuki, Nucl. Phys. B 38, 54 (1995).

6. Y. Suzuki, talk presented at Neutrino '98 (Takayama, Japan, June, 1998).

7. T. Kajita, talk presented at Neutrino '98 (Takayama, Japan, June, 1998).

8. J. N. Bahcall, S. Basu, and M. H. Pinsonneault, Phys. Lett. B 433, 1 (1998).

9. A. S. Brun, S. Turck-Chieze, and P. Morel, Ap. J. 506, 913 (1998) and private communication; S. Turck-Chieze and I. Lopez, Ap. J. 408, 347 (1993).

10. This section taken from W. C. Haxton, nucl-th/9901037.

11. S. M. Austin, N. Anantaraman, and W. G. Love, Phys. Rev. Lett. 73, 30 (1994); J. W. Watson et al., Phys. Rev. Lett. 55, 1369 (1985).

12. K. Lande, talk presented at Neutrino '98 (Takayama, Japan, June, 1998).

13. J. N. Bahcall, Neutrino Astrophysics, (Cambridge University, Cambridge, 1989).

14. C. W. Johnson, E. Kolbe, S. E. Koonin, and K. Langanke, Ap. J. 392 , 320 (1992).

15. B. W. Filippone, A. J. Elwyn, C. N. Davids, and D. D. Koetke, Phys. 
Rev. Lett. 50, 412 (1983).

16. J. N. Bahcall and R. Ulrich, Rev. Mod. Phys. 60, 297 (1988).

17. J. N. Bahcall and W. C. Haxton, Phys. Rev. D 40, 931 (1989).

18. V. Castellani, S. Degl'Innocenti, G. Fiorentini, M. Lissia, and B. Ricci, Phys. Rev. D 50, 4749 (1994).

19. J. N. Bahcall and R. Davis, Jr., in Essays in Nuclear Astrophysics, ed. C. A. Barnes, D. D. Clayton, and D. Schramm (Cambridge Univ. Press, Cambridge) p. 243.

20. F. W. W. Dilke and D. O. Gough, N 240, 262 (1972).

21. W. J. Merryfield, in Solar Modeling, ed. A. B. Balantekin and J. N. Bahcall (World Scientific, Singapore, 1995).

22. A. Cumming and W. C. Haxton, Phys. Rev. Lett. 77, 4286 (1996).

23. N. Hata and P. Langacker, Phys. Rev. D 56, 6107 (1997).

24. K. M. Heeger and R. G. H. Robertson, Phys. Rev. Lett. 77, 3720 (1996).

25. For a proper (wave packet) derivation see M. Nauenberg, submitted to Phys. Lett. B.

26. S. P. Mikheyev and A. Smirnov, Sov. J. Nucl. Phys. 42, 913 (1985); L. Wolfenstein, Phys. Rev. D 17, 2369 (1979).

27. H. Bethe, Phys. Rev. Lett. 56, 1305 (1986).

28. W. C. Haxton, Phys. Rev. Lett. 57, 1271 (1986).

29. S. J. Parke, Phys. Rev. Lett. 57, 1275 (1986).

30. C. S. Lim and W. J. Marciano, Phys. Rev. D 37, 1368 (1988).

31. E. Kh. Akhmedov, Sov. J. Nucl. Phys. 48, 382 (1988).

32. G. Raffelt, Phys. Rev. Lett. 64, 2856 (1990).

33. K. Fujikawa and R. E. Shrock, Phys. Rev. Lett. 43, 963 (1980).

34. W. C. Haxton and G. J. Stephenson, Jr., Prog. Part. Nucl. Phys. 12, 409 (1984).

35. A. Mezzacappa et al., Ap. J. 495, 911 (1998); H.-Th. Janka and E. Muller, Astron. Astrophys. 306, 167 (1996); A. Burrows, S. Hayes, and B. A. Fryxell, Ap. J. 450, 830 (1995).

36. S. E. Woosley and W. C. Haxton, Nature 334, 45 (1988); S. E. Woosley, D. H. Hartmann, R. D. Hoffman, and W. C. Haxton, Ap. J. 356, 272 (1990).

37. F. X. Timmes, S. E. Woosley, and T. A. Weaver, Ap. J. Suppl. 98, 617 (1995).

38. J. J. Cowan et al., astro-ph/9808272 (to appear in $A p$. J).

39. S. E. Woosley, J. R. Wilson, G. J. Mathews, R. D. Hoffman, and B. S. Meyer, Ap. J. 433, 229 (1994).

40. W. C. Haxton, K. Langanke, Y.-Z. Qian, and P. Vogel, Phys. Rev. Lett. 78, 2694 (1997) and Phys. Rev. C 55, 1532 (1997). 
41. Y.-Z. Qian et al., Phys. Rev. Lett. 71, 1965 (1993). 